\title{
P.BEROL. INV. 14081. FRAMMENTO DI UNA NUOVA COPIA DEL DIGESTO DI ETÀ GIUSTINIANEA ${ }^{s}$
}

\author{
Nota dei m.e. MARCO FRESSURA (*) e DARIO MANTOVANI (**)
}

(Adunanza del 9 marzo 2017)

SunTO. - Edizione riveduta e aggiornata di un frammento di codice papiraceo del $D i$ gesto di Giustiniano, recentemente identificato. Il codice, riferibile al VI sec., faceva verosimilmente parte di un'edizione dell'opera articolata in più tomi. Si tratta di una scoperta che tende a confermare il quadro di un'immediata e ampia circolazione del Digesto nel contesto scolastico e della pratica del diritto.

$$
* * *
$$

ABSTRACT. - Updated edition of a recently discovered fragment of Justinian's Digest. The fragment comes from a sixth-century papyrus codex that in all likelihood was part of an edition of the Digest in multiple tomes. In addition to providing palaeographical and codicological information, this new finding supports the idea that the Digest had considerable circulation and was used in legal education and legal practice.

(*) Università degli Studi Roma Tre, Roma, Italia.

E-mail: marco.fressura@uniroma3.it.

(**) Collège de France, Parigi, Francia.

E-mail: dario.mantovani@college-de-france.fr.

$\checkmark$ The research leading to these results has received funding from the European Research Council under the European Union's Seventh Framework Programme (FP7/2007-2013) / ERC grant agreement nr. 341102 'Redhis. Rediscovering the hidden structure. A new appreciation of Juristic texts and Patterns of thought in Late Antiquity', Università degli Studi di Pavia (Dipartimento di Giurisprudenza), Principal Investigator Dario Mantovani, Senior Staff Luigi Pellecchi (<http://redhis.unipv.it $>)$. - Il presente contributo riprende, con aggiornamenti e correzioni, Fressura-Mantovani 2017. Si devono a Dario Mantovani i $\$ \mathbb{S} 1$, 4-6, a Marco Fressura i $\$ S$ 2-3. La validità dei riferimenti a risorse in rete è stata verificata nell'ottobre 2019. 


\section{PRemessa}

Nel quadro del progetto Redhis, che studia la circolazione delle opere di giuristi romani classici fra III e VI sec., è stata effettuata un'ampia esplorazione di pergamene e papiri latini e greci conservati nelle collezioni europee ed extra-europee. Si è così voluto accertare, censendo le testimonianze materiali, se e in che misura il diritto romano classico abbia rappresentato un elemento costitutivo del diritto tardoantico. Quest'esplorazione ha portato alla luce un numero cospicuo di testimoni inediti di opere di giuristi classici e di commenti ad esse, oltre a consentire una serie di letture migliorative di testi già editi. Esauritasi nel corso del III sec. la produzione di nuove opere, la letteratura giurisprudenziale (specialmente quella compresa fra l'età degli Antonini e l'età dei Severi) ha continuato ad essere copiata e utilizzata - come mostrano glosse e commenti anch'essi ben rappresentati nel campione dei testi reperiti - costituendo una sorta di 'struttura nascosta' del diritto romano anche nella Tarda Antichità e preparando le condizioni culturali per la compilazione giustinianea.

Nel corso della ricognizione delle collezioni papirologiche sono stati rinvenuti anche altri frammenti di contenuto giuridico, cioè diversi dalle opere dei giuristi classici (o da commenti alle opere stesse). In ragione del loro contenuto, essi non sono perciò destinati ad essere inclusi nella raccolta delle pergamene e papiri giurisprudenziali pregiustinianei cui è rivolto in modo specifico il progetto Redhis. In alcuni casi si tratta di frammenti di notevole interesse, che costituiscono risultati in sé della ricerca, di cui pare opportuno perciò dare immediata notizia, come nel caso di P.Berol. inv. 14081, in cui M. Fressura ha identificato un nuovo testimone del Digesto di Giustiniano, risalente all'età giustinianea. ${ }^{1}$ Il frammento di questa nuova copia, oltre a consentire osservazioni e confronti di carattere grafico e codicologico, porta argomenti a favore dell'effettiva circolazione e uso del Digesto nella scuola e nella prassi.

1 Rendeva nota l'esistenza del frammento, senza ancora un'identificazione, Ammirati 2015, 98-99. Riproduzione in Berliner Papyrusdatenbank, <http://berlpap.smb .museum/06296/>. 


\section{Descrizione PALEOgRafica e Codicologica}

Il frammento di foglio di codice papiraceo P.Berol. inv. 14081 $(\mathrm{TM}=\mathrm{LDAB} 828809)$ fu acquisito sul mercato antiquario egiziano da Carl Reinhardt, dragomanno presso il consolato generale tedesco del Cairo (1893-1896), ed entrò a far parte della Papyrussammlung dell'Ägyptisches Museum di Berlino probabilmente nel 1896, insieme agli altri cimeli della Sammlung Reinhardt. ${ }^{2}$

Il papiro, che misura mm 140 (larghezza) $\times 110$ (altezza), costituisce porzione dell'angolo superiore esterno del foglio. Su entrambi i lati, la maggior parte della superficie è occupata dai margini superiore $(\mathrm{mm} \mathrm{67)}$ ed esterno $(\mathrm{mm} 85)$, che sembrano conservarsi nelle dimensioni originarie e non recano tracce di scrittura. Lo specchio scrittorio rimanente, su ciascuna pagina, copre ca. mm 55 (larghezza) $\times 40$ (altezza) e mostra le prime cinque righe di testo, mutile al principio (lato $\rightarrow$ ) e alla fine (lato $\downarrow$ ). Recto $(\rightarrow$ ) e verso $(\downarrow)$ del foglio si determinano grazie all'identificazione del contenuto rispettivamente con D. 6.1.73-74 (Ulp. 17 ad ed.; Paul. 21 ad ed.) e 6.1.77 (Ulp. 17 ad ed.). In base al testo è possibile ricostruire uno spazio scrittorio sviluppato sulla piena pagina, largo ca. mm 150 e articolato su 30 righe (di 23-31 lettere nella sezione superstite ${ }^{4}$ ), per un'altezza complessiva di ca. $\mathrm{mm} 267$, dati modulo della scrittura di $\mathrm{mm} 5$ e un'interlinea di

2 Gli inventari dell'Ägyptisches Museum di Berlino non riportano informazioni circa la data di acquisizione di P.Berol. inv. 14081, ma, là dove sia stato registrato, l'a. 1896 ricorre per tutti gli altri frammenti provenienti dalla Sammlung Reinhardt. Documenti riguardanti l'incorporazione di quest'ultima nella Papyrussammlung non si ritrovano presso il Museo, presumibilmente esistiti ma perduti nel corso della Seconda Guerra Mondiale (Marius Gerhardt, ÄMP, per litteras, 3 settembre 2015). Un dettagliato profilo di Carl Reinhardt (1856-1903) è tracciato da Nagel 2014, 1.

3 La combinazione e l'ordine dei testi conservati porta a escludere che il manoscritto sia testimone della tradizione diretta delle opere giurisprudenziali identificate.

4 Non significativa per il computo r. 2 recto, che conclude D. 6.1 .73 e occupa solo una parte dello spazio orizzontale disponibile fino al margine destro dello specchio scrittorio. 
$\mathrm{mm} \mathrm{4.}{ }^{5}$ Considerati i margini superiore ed esterno ancora conservati, se si ipotizzano un margine inferiore almeno equivalente al superiore, e un margine interno ampio ca. la metà di quello esterno ( $\mathrm{mm} \mathrm{45),} \mathrm{ci}$ si approssima a un formato originario di dimensioni non inferiori a mm 280 (larghezza) $\times 401$ (altezza).$^{6}$

È probabile che il codice sia stato allestito in tempi non lontani dal terminus post quem implicato dall'identificazione del contenuto (a. 533), come suggerisce la scrittura testuale, un'onciale nella tipizzazione BR, attestata da manoscritti di mano orientale e argomento prevalentemente giuridico riferiti di norma al V-VI sec. ${ }^{7}$ Se si eccettua un intervento di correzione a r. 2 recto, effettuato da seconda mano, quanto resta appare vergato da un unico scriba professionale. Tra le particolarità della scrittura sono da notare: il rispetto dell'uso latino nell'applicazione di un chiaroscuro obliquo alle lettere tondeggianti $(c, e, o)$ e nel tracciato di $n$ (tratti verticali sottili; tratto obliquo media-

5 mm 261 (29 righe più interlinea) + 6 (prima o ultima riga priva di interlinea inferiore o superiore).

6 Group 1 ('The Largest Sizes: Very Broad') di Turner 1977, 14. Trattandosi di un frammento del settore esterno del foglio, il breve intervallo testuale fra recto e verso non induce di per sé a escludere che il manoscritto presentasse in origine due colonne per pagina; nel caso particolare, tuttavia, l'eventualità si può ritenere improbabile non solo considerato quanto si ricostruisce per altri frammenti papiracei del Digesto già noti (vd. più avanti), ma visto anche che, in quel modo, la larghezza del formato di P.Berol. inv. 14081 supererebbe l'altezza (ipoteticamente, in aggiunta a quanto ricostruito, $\mathrm{mm} 150$ più un intercolumnio di ampiezza simile al margine interno), con un rapporto $l / h>1$. Stando a Turner 1977, 14-22, 26-30, Menci 1997, 685-686, tale caratteristica risulta estremamente rara nell'ambito dei codici papiracei e pergamenacei. Fra i testimoni papiracei greci, essa è stata attribuita almeno a P.Oxy. XX 2258 (VI sec.; Call.; LDAB 523; TM 59424; MP $3^{3}$ 0186), ma la questione si presenta dubbia: Turner 1977, 14, 84, $103 \mathrm{n}^{\circ} 28$, ricostruisce le dimensioni in $\mathrm{mm} 370 \times 280$, ma Maehler 2000, 34, e Irigoin 2009, 74, fatto salvo il valore della larghezza, stimano l'altezza originaria del codice rispettivamente in mm 450 e 400; Porro 2011, 314-315, invece, riabbassa il valore dell'altezza a mm 310, senza escluderne uno maggiore, ma non superiore al valore della larghezza (sempre mm 370), per un formato complessivo «quasi quadrato».

7 In sintesi, Cherubini-Pratesi 2010, 98-100; ulteriori contributi in Ammirati 2010, 84-88, 90-97, Cavallo-Fioretti 2014, 54-56, Ammirati 2015, 94-99. 
no spesso) $;{ }^{8}$ il tracciato di $t$, con tratto orizzontale di sporgenza breve a sinistra, brevissima a destra. $N$ in principio di r. 6 verso, di dimensioni doppie rispetto alla scrittura testuale, è ingrandita in quanto prima lettera della pagina ${ }^{9}$ e, come certifica la ricostruzione del testo, non segnala una partizione testuale (in tal caso si sarebbe verosimilmente presentata in ekthesis); il fenomeno è ben testimoniato nei più antichi codici latini ${ }^{10}$ e si riscontra per es., sebbene con discontinuità, nel caso della prima lettera di ciascuna delle due colonne per pagina nel 'Codex Florentinus' del Digesto $(\mathbf{F}),{ }^{11}$ dove si concretizza in un'alternanza di forme onciali e capitali. Nel caso di P.Berol. inv. 14081, il tracciato ancipite di $n$ iniziale di pagina impedisce una rigorosa classificazione della scrittura distintiva adoperata, che nel manoscritto integro - per la realizzazione di altre lettere ingrandite e degli apparati paratestuali - poteva presentarsi in forme onciali coerenti con la scrittura testuale $\mathrm{o}$, con altrettanta probabilità, sotto la specie di una capitale libraria più o meno ibridata con l'onciale stessa, similmente a quanto avviene, ancora, in $\mathbf{F}$. $^{12}$

Come risulta dalla ricostruzione complessiva del formato e dell'assetto testuale, la pagina recto di P.Berol. inv. 14081 allo stato pristino era ca. 3,6 volte meno capiente della corrispondente pagina in $\mathbf{F}\left(\mathrm{I} \mathrm{f.} 117^{\mathrm{r}}\right)$. Ammesso che tale proporzione si mantenesse costante o non variasse significativamente, il testo del Digesto precedente il frammento - compresi i materiali accessori anteposti al libro primo -

8 Lowe 1961, 283.

9 Ovvero colonna di scrittura, in questo caso singola.

10 Lowe 1925, 202-205.

11 Firenze, Biblioteca Medicea Laurenziana, s.n. (paulo post 16 dicembre 533; cod. perg.; LDAB 7619; TM 66369; CLA III 295), che costituisce il solo esemplare integro dell'opera datato al VI sec. Vd. almeno Baldi 2010 e Ammirati 2015, 96 (anche per ulteriore bibliografia); riproduzione integrale in Pandette 1902-1910, e CorbinoSantalucia 1988. Per le modalità di applicazione dell'ingrandimento della lettera iniziale di colonna in F, Kaiser 2001, 140-141.

12 Per l'esame sistematico di questo genere di dettagli, F resta l'esemplare integro di riferimento. Sulla scrittura cui si fa cenno, Cavallo 1996, 26; sugli apparati paratestuali nei manoscritti di contenuto giuridico, Mantovani 2015, Mantovani 2018, 241-284. 
doveva essere contenuto in ca. 420 fogli, per un'edizione complessiva che, se effettivamente portata a termine, raggiungeva i 3200 fogli di consistenza. ${ }^{13}$ Una tale edizione doveva essere certamente realizzata impegnando l'opera di più scribi ${ }^{14} \mathrm{e}$, soprattutto, doveva essere articolata in un certo numero di tomi. Supponendo questo, è verosimile ritenere che il papiro non provenga dal primo della serie, ma almeno dal secondo. ${ }^{15}$

Per dimensioni originarie e caratteristiche editoriali, P.Berol. inv. 14081 trova confronto puntuale in altri tre frammenti di codici papiracei testimoni di porzioni del Digesto, tutti allestiti in onciale BR (modulo delle scritture: mm 4-5) e riferibili al VI sec. Due di essi provengono dall'Egitto: P.Heid. inv. L 4 (D. 5.2.17-19, con marginalia greci) ${ }^{16} \mathrm{e}$

13 Il foglio recto di P.Berol. inv. 14081, al suo stato integro, conteneva testo per 789-792 lettere complessive, fatti salvi eventuali errori dello scriba e varianti testuali che, ragionevolmente, non potevano incidere in modo significativo sul computo attuale, basato su Mommsen 1870; la pagina corrispondente in $\mathbf{F}\left(\mathrm{I} \mathrm{f.} 117^{\mathrm{r}}\right)$ contiene 2828 lettere, con una proporzione di 3,57. Per il calcolo approssimativo della consistenza dell'edizione integrale secondo il formato testimoniato dal papiro, si moltiplica tale valore per il numero complessivo di 901 fogli scritti pertinenti al Digesto in F (I 438,5 + II 462,5).

14 Dalle tredici alle quindici mani diverse hanno operato in F: Baldi 2010, 113-114

15 Ammesso che esista e sia dimostrabile un qualche legame tra le partes tematiche del Digesto, comprendenti gruppi di libri, e l'allestimento materiale dei testimoni manoscritti, P.Berol. inv. 14081, in quanto attesta il libro 6 e si inquadra nella pars secunda de indiciis (libri 5-11), potrebbe collocarsi intorno a f. 73 di un eventuale secondo tomo che contenesse almeno i libri 5-6. Sul piano codicologico - in rapporto con un eventuale primo tomo di ca. 340 fogli - l'ipotesi non appare implausibile, sebbene Turner 1977, 82-83, riporti un valore massimo attestato di ca. 300 fogli per la consistenza del codice papiraceo. Sulla questione delle partes in relazione con l'assetto di F, Stolte 1984, Kaiser 2001, 162-168.

16 LDAB 2557; TM 61413; MP3 2966; CLA VIII 1221; CPL 87. Il papiro, già noto come P.Heid. inv. 1272, consiste di due frammenti contigui di un foglio di codice. Edizione in Gerhard 1907; per il testo dei marginalia, Gradenwitz 1902, 458-459, Gerhard-Gradenwitz 1903, McNamee 2007, 497-499. L'attribuzione del papiro al Digesto è stata messa in dubbio da Boyé 1926, 481-488, che vi vedrebbe piuttosto un esemplare della tradizione diretta di Paul. 2 quaest. (cf. Collinet 1925, 283), ma a ciò si oppone l'evidenza di un fatto, che lo stesso Boyé 1926, 486, prendeva in considerazione, 
P.Ryl. III 479 + 480 (D. 28.8.11, 30.11-13, 19, 22-26); ${ }^{17}$ il terzo, P.Pommersf. L 1-6 (D. 45.1.35-36, 38, 41-46, 48-49, 72-73), ${ }^{18}$ risulta dallo smantellamento di un'antica legatura libraria realizzata in cartone, ${ }^{19}$ quest'ultimo fabbricato con materiali papiracei di scarto provenienti da una località dell'Italia romana, probabilmente Ravenna.$^{20} \mathrm{Le}$ caratteristiche dei papiri si possono così riassumere:

ritenendolo a torto non risolutivo (cf. Wenger 1950, 137-143, Wenger 1953, 592-593 nt. 143): quanto resta delle ultime quattro righe al recto è compatibile con il testo di Paul. $l$. s. de inoff. testam. D. 5.2.18 e in più, all'estremità dell'ultima riga, si scorge anche il segno di punteggiatura (:), che chiude il caput coerentemente con quanto tràdito, per il Digesto, da $\mathbf{F}$ (I f. $102^{\mathrm{v}}$, con simile segno di punteggiatura di funzione analoga). Una descrizione dei frammenti anche in Seider 1981, 110-112 n 40, Ammirati 2015, 97.

17 P.Ryl. III 479: LDAB 2554; TM 61410; MP³ 2967; CLA Suppl. 1723; CPL 89. P.Ryl. III 480: LDAB 5982; TM 64748; MP33 2980; CLA Suppl. 1724. P.Ryl III 479 consiste di cinque frammenti (a-e) riconducibili ad almeno due fogli di codice. Edizione in Roberts 1938, 85-90, con attribuzione solo per i frr. a (D. 30.11-13) e b (D. 30.22-26); a rettifica di Schulz 1941, terza edizione, non ancora soddisfacente, in Düll-Seidl 1941, con attribuzione dei frr. c, d (D. 30.19, 24), e (D. 30.8, 10: quest'ultima impossibile, poiché in tal modo il fr. e $\rightarrow$ dovrebbe trovarsi su una stessa pagina con il fr. a $\downarrow$ e il fr. e $\downarrow$ addirittura sulla pagina precedente). In occasione del XXIX Congresso Internazionale di Papirologia (Lecce, 28 luglio-3 agosto 2019) ho dedicato a P.Ryl. III 480 la relazione Un nuovo frammento papiraceo del Digesto: problemi di ricostruzione e trasmissione testuale, proponendo identificazione, attribuzione ed edizione del papiro. Descrizione di P.Ryl. III 479 anche in Seider 1981, 105-107 n 37, Ammirati 2015, 95-96; riproduzione in Rylands Papyri Collection, <http://luna.manchester.ac.uk/luna/servlet/Manchester Dev 93 3>; su P.Ryl. III 480, Ammirati 2015, 90.

18 LDAB 2556; TM 61412; CLA IX 1351. Il papiro consiste di sette fogli frammentari di codice (f. VII è diviso in tre frammenti distaccati; $f$. VI è perduto). 'Apografo' e annotazioni in Mommsen 1870, XXXX-XXXXI, LXXXXII-LXXXXIII, $11^{*}-16$, compreso $\mathrm{f}$. VI già allora irreperibile, per il testo del quale fa fede Zachariä 1842 , 263-265. Vd. anche Herrmann 1966, 190, 192, Seider 1981, 102-105 n 36, Sirks-SijpsteijnWorp 1996, 137-142 (con Stolte 1999a, 115-119, Stolte 1999b), Ammirati 2015, 95.

19 Sirks-Sijpsteijn-Worp 1996, 5-8, 18-23. Per una descrizione generale del fenomeno, fra gli altri, Szirmai 1999, 28-31.

20 Tjäder 1958, 37-41; cf. Herrmann 1966, 189, Sirks-Sijpsteijn-Worp 1996, 17 18, 23-31, Ammirati 2015 95, mentre Cavallo 1978, 234, colloca a Ravenna la produzione stessa del manoscritto. La presenza (o confezione) dei materiali in quella o altra località d'Italia si riferisce al periodo della riconquista giustinanea, forse anche a qualche anno prima della pragmatica sanctio del 554 (del resto, le operazioni militari si protrassero fino al 562: per es., Cameron - Ward-Perkins - Withby 2000, 82, 534); vd., più avanti, $\mathbb{\$} 6$. 


\begin{tabular}{lccc} 
& $\begin{array}{c}\text { FORMATO } \\
(\mathrm{mm})\end{array}$ & $\begin{array}{c}\text { SPAZIO SCRITTORIO } \\
(\mathrm{mm})\end{array}$ & $\begin{array}{c}\text { RIGHE PER } \\
\text { PAGINA }\end{array}$ \\
\hline P.Berol. inv. 14081 & $280 \times 401$ & $150 \times 267$ & 30 \\
P.Heid. inv. L 4 $4^{21}$ & $301 \times 361$ & $170 \times 221$ & 28 \\
P.Ryl. III 479 + 480 & $335 \times 416$ & $200 \times 276$ & 35 \\
P.Pommersf. L 1-6 & $282 \times 363$ & $160 \times 237$ & 30 \\
\hline
\end{tabular}

Nonostante la condivisa provenienza geografica, le significative ancorché lievi differenze fra i connotati codicologici ricostruiti per le tre testimonianze egiziane $e^{24}$ non inducono a riferire i diversi frammen-

21 Plausibile la ricostruzione di 28 righe per pagina in Gerhard 1907, in base alle quali, data una riga più interlinea di $\mathrm{mm} 8$, si ottiene l'altezza dello spazio scrittorio, mentre la larghezza si stima approssimativamente sulla quantità di lettere contenute nelle righe ricostruite. Considerata la misura di mm 86 del margine esterno, pressoché identica a quella di P.Berol. inv. 14081, in base ai mm 67 del margine superiore di quest'ultimo si assegna il valore approssimato di $\mathrm{mm} 70$ ai margini superiore e inferiore di P.Heid. inv. L 4. Un margine interno di $\mathrm{mm} 45$ si ricava per approssimazione dai $\mathrm{mm}$ 42 di P.Pommersf. L 1-6.

2232 righe per pagina secondo Roberts 1938, 86, ma 34 righe secondo DüllSeidl 1941 e 36 righe secondo Kaiser 2004, 404, stime più vicine al valore di 35 righe che a me pare preferibile. Plausibile il valore di mm 200 calcolato da Roberts 1938, 86, per la larghezza dello spazio scrittorio, mentre il valore dell'altezza, mm 220, dev'essere corretto al rialzo, tenendo conto delle 3 righe aggiunte alla ricostruzione (riga più interlinea ca. $\mathrm{mm}$ 8). Nessun margine si conserva intero; si assegnano perciò i valori ipotetici di: $\mathrm{mm} 70$ ai margini superiore e inferiore, per approssimazione ai $\mathrm{mm} 67 \mathrm{del}$ margine superiore di P.Berol. inv. 14081 e ai mm 62 del margine inferiore di P.Pommersf. L 1-6; mm 45 al margine interno, per approssimazione ai $\mathrm{mm} 42 \mathrm{di}$ P.Pommersf. L 1-6; mm 90 per il margine esterno, per approssimazione ai mm 86 di P.Heid. inv. L 4. Il risultato complessivo si allontana dai $\mathrm{mm} 260 \times 320$ calcolati da Roberts 1938, 86, per le dimensioni originarie del foglio.

23 Mommsen 1870, 11*: «folium nullum hodie integrum est: at cum in foliis primo secundo septimo adsit margo inferior, computando effectum est singulas paginas habuisse uersus ad tricenos, singulos uersus elementa ad uicena octona». Confermato tale risultato (30 righe) e considerati i mm 8 di riga più interlinea si ottiene l'altezza dello spazio scrittorio, mentre la larghezza si stima approssimativamente in base alla quantità di lettere contenute nelle righe ricostruite $(\mathrm{mm} 170 \times 245$ in CLA IX 1351). Conservati il margine interno ( $\mathrm{mm} \mathrm{42)}$ e inferiore ( $\mathrm{mm} \mathrm{63)}$; si ipotizzano un margine superiore ampio almeno quanto l'inferiore e un margine esterno ampio ca. due volte l'interno ( $\mathrm{mm} \mathrm{80}$ ).

24 Il codice papiraceo frammentario P.Sorb. inv. 2219 (VI sec.; LDAB 2555; TM 
ti a un'impresa editoriale unitaria; $;^{25}$ P.Berol. inv. 14081 si inserisce così coerentemente nel quadro di una diffusione del Digesto, probabilmente precoce ed ecumenica, ${ }^{26}$ in edizioni composte di più tomi papi-

61411; MP3 2971; CLA Add. I 1858; CPL 100), riferibile a D. 19, non è stato considerato nella presente analisi poiché la sua scrittura (minuscola ogivale inclinata) non è congruente con quella degli altri esemplari papiracei noti. Edizioni di P.Sorb. inv. 2219, complete dei marginalia greci, sono in de Ricci 1912, Scheltema 1958, Scheltema 1970, McNamee 2007, 499-500; un severo commento è stato riservato al contributo di Naber 1935 da parte di van der Wal 1979, 275-276. Per una descrizione del papiro, vd. anche Seider 1981, 68-69 $\mathrm{n}^{\circ}$ 18, Ammirati 2015, 99. Riproduzione parziale in Institut de Papyrologie de la Sorbonne. Le Collections de Papyrus. Papyrus Latins, $<$ http://www.papyrologie.paris-sorbonne.fr/menu1/ collections/platin.htm>. Alcune rettifiche al contenuto di questa nota, particolarmente riguardo a una più corretta attribuzione del numero di inventario ai frammenti (cf. Fressura-Mantovani 2017, 695 696 nt. 24), si deve a un'autopsia effettuata dagli autori presso l'Institut de papyrologie de la Sorbonne (Parigi) il 13 aprile 2018.

25 Per un ragionamento in tal senso, invece, le pur evidenti differenze di mano risultano irrilevanti, poiché - come già osservato a testo - si presuppone che la realizzazione di un'edizione integrale del Digesto richiedesse comunque una divisione del lavoro di copia fra più scribi.

26 Delle tre testimonianze egiziane, solo P.Ryl. III $479+480$ è forse riconducibile all'area più circoscritta della Tebaide - se può far fede l'acquisto effettuato a Luxor (1917), del quale riferisce Roberts 1938, 64 (cf. Elliott 1999, 12-13) - ma questa informazione, anche se messa in rapporto con le caratteristiche materiali del papiro, non basta per affermare, con Lanata 1984, 23, che l'originaria edizione «avrebbe potuto essere la copia ufficiale inviata al governatore», ipotesi che inoltre non individua chiaramente a quale specie dovrebbe appartenere il 'governatore' destinatario (diverse condizioni si presenterebbero presupponendo un invio precedente o seguente la riforma amministrativa dell'area egiziana del 539); vd. Demicheli 2000, Palme 2007. A una provenienza italiana, forse ravennate, di P.Pommersf. L 1-6 si è già fatto cenno; dovette circolare in Italia anche l'antigrafo del ms. Napoli, Biblioteca Nazionale "Vittorio Emanuele III", IV A 8, scriptio inf. ff. 36-39 (D. 10.2.3-16; 23-29; 10.4.12-19), codice pergamenaceo in onciale non tipizzata (VI sec.; LDAB 7754; TM 66506; CLA III 402), che riproduce apparentemente le modalità di presentazione del testo tipiche di un modello papiraceo (piena pagina di 32 righe; vd. anche, più avanti, $\mathbb{S} 5$ 5-6). In nessun caso, tuttavia, si può individuare il luogo di allestimento dei manoscritti in questione, dato che essi rappresentano realizzazioni singolari tutte ugualmente fedeli a un modello editoriale consolidato, probabilmente concepito in ambito orientale ma che proprio le condizioni di esistenza di P.Pommersf. L 1-6 impediscono di considerare con certezza peculiare del solo Egitto; questo d'altra parte non significa che l'Egitto stesso non possa essere il luogo della più remota origine 
racei $^{27}$ di grande formato, ${ }^{28}$ che potevano arrivare a includere tutti i 50 libri dell'opera. ${ }^{29}$ Una circolazione di altre opere del Corpus iuris ciuilis, con simili caratteristiche codicologiche e paleografiche (in particolare, l'uso dell'onciale BR), è peraltro testimoniata dai codici papiracei P.Oxy. XV 1814, recante un indice relativo alla prima edizione del Codex (a. 529-534), ${ }^{30}$ e PSI XIII 1347, pertinente al libro settimo del Codex repetitae praelectionis (post 534, ma sempre entro il VI sec.). ${ }^{31}$ L'opzione per un formato molto grande del codice papiraceo (ca. $\mathrm{mm}$ $300 \times 400$ ), con la superficie complessiva delle aree marginali di ca. $1,5-2$ volte più ampia dello spazio scrittorio sulla pagina, non costitui-

di P.Pommersf. L 1-6, data la possibilità che, fatta salva la provenienza italiana, anche questo manoscritto sia dovuto a manodopera di educazione orientale e abbia circolato in ambiente ellenofono (Ammirati 2015, 95).

27 Non è pensabile che P.Pommersf. L 1-6 provenga da un'edizione articolata in due tomi analogamente a F, come pure ipotizzano Sirks-Sijpsteijn-Worp 1996, 17: in primo luogo, non diversamente da quanto stimato qui per P.Berol. inv. 14081, già Mommsen 1870, 11*, aveva ricostruito, in base ai frammenti di Pommersfelden, un'edizione originaria estesa su più di 3000 fogli papiracei, che appare inverosimile potessero essere divisi solo in due parti di 1500 fogli ciascuna (vd., supra, nt. 15); in secondo luogo, circa la rilegatura di $\mathbf{F}$ in due tomi si dispone di documentazione certa solo a partire dal XV sec. e nulla mi risulta si conosca a proposito della più remota articolazione dell'edizione. Su quest'ultimo aspetto, Stolte 1984, 71-72, 88, e soprattutto Baldi 2010, 110, 132, 153.

28 Si tratta di una realizzazione peculiare del «new format» cui allude $\mathrm{McNamee}$ 2007, 14, 79-80; vd. anche Ammirati 2015, 97-98.

29 È lecito supporlo in base all'attestazione nei papiri di più libri dell'opera distanti fra loro e abbastanza ben distribuiti: 5, 6, 28, 30, 45, ai quali si aggiungono il libro 10 (antigrafo del ms. Napoli, Biblioteca Nazionale "Vittorio Emanuele III", IV A 8; vd., supra, nt. 26) e il libro 19 (P.Sorb. inv. 2219). Vd. anche, più avanti, $\mathbb{} 6$.

30 LDAB 6324; TM 65083; MP3 2969; CLA Suppl. 1713; CPL 101; AmelottiMigliardi Zingale 1985, 17-23 n 1. Vd. anche Corcoran 2008, 73-111, Ammirati 2015, 95. Il papiro consiste di più frammenti di foglio di formato complessivo non inferiore a $\mathrm{mm}$ $223 \times 344$, con 30 (recto)-28 (verso) righe per pagina e modulo della scrittura di mm 4-5. Riproduzione in POxy: Oxyrbynchus online, <http://www.papyrology.ox.ac.uk/ POxy/>.

31 LDAB 6272; TM 65031; MP3 2970; CLA III 293; CPL 99; Amelotti-Migliardi Zingale 1985, 27-31 $\mathrm{n}^{\circ}$ 3; Ammirati 2015, 95. Il papiro consiste di cinque frammenti di un foglio; significativo il numero di 30 righe per pagina, il modulo della scrittura di $\mathrm{mm}$ 4-5, la misura di una riga più interlinea di $\mathrm{mm} 8$ e il valore del margine inferiore di almeno mm 64-66. Riproduzione in PSIonline, <http://www.psi-online.it>. 
sce tuttavia un'innovazione connessa con l'opera di codificazione giustinianea, ma si colloca in continuità con pratiche editoriali già sperimentate, almeno in Oriente, nei decenni a essa precedenti - risalendo verosimilmente fino al $\mathrm{V}$ sec. $^{32}$ - per la pubblicazione della letteratura giurisprudenziale e, più in generale, giuridica. ${ }^{33}$ Fra i reperti latini, è peculiare P.Vindob. L $110,{ }^{34}$ che tramanda un testo di diritto romano non ancora identificato, probabilmente pregiustinianeo, ${ }^{35}$ e costituisce frammento di un foglio di codice papiraceo vergato anch'esso in onciale BR (modulo della scrittura: mm 5), caratterizzato da spazio marginale di notevole ampiezza: mm 87-91 per il margine esterno,

32 Manoscritti giuridici di grande formato sono attestati già per il IV sec. almeno in forma pergamenacea, come nel caso di P.Berol. inv. 6757 (LDAB 4133; TM 62941; $\mathrm{MP}^{3}$ 2985; CLA VIII 1033), frammento di una trattazione giurisprudenziale latina de xiudiciis contenuta in un codice, impaginato a due colonne, che raggiungeva verosimilmente i $300 \mathrm{~mm}$ di larghezza; vd. Ammirati 2015, 88. Sempre per il IV sec., è lecito menzionare la testimonianza di Libanio, che descrive studiosi di diritto romano alle

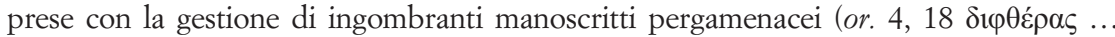

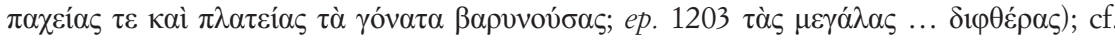
Cribiore 2007, 188.

$33 \mathrm{Vd}$., supra, nt. 28. La notevole estensione della superficie marginale garantiva non solo longevità a manoscritti che, per il loro contenuto tecnico, erano probabilmente consultati di frequente (eventuali ripetuti restauri potevano comportare successive rifilature), ma forniva anche ampio spazio per l'inserimento di scrittura accessoria. L'adozione immediata di un formato con questa caratteristica per le opere del Corpus iuris è soprattutto significativa in rapporto con i manoscritti del Digesto, sui margini dei quali era eventualmente lecito apporre solo speciali tipi di annotazione, con l'esclusione di commentarii interpretativi del testo; su queste limitazioni, imposte dall'imperatore Giustiniano, Falcone 2014, con bibliografia.

34 LDAB 6397; TM 65155; MP3 2984; CLA X 1538; CPL 245. Quasi nulla resta dello spazio scrittorio, salvo le estremità di alcune righe, ma sono interamente leggibili due marginalia latini (uno per ciascuna pagina), per il testo dei quali vd. Girard-Senn 1967, 472. Riproduzione in Österreichische Nationalbibliothek, Katalog der Papyrussammlung, $<$ https://www.onb.ac.at/bibliothek/sammlungen/papyri/bestaende/bestandsrecherche/>.

35 La presenza di abbreviazioni rese secondo il sistema delle notae iuris e l'apparente estraneità delle sequenze testuali recuperabili rispetto al Corpus iuris fanno pensare a un'edizione precedente la codificazione giustinianea; il contenuto dei marginalia può suggerire l'identificazione del testo con un trattato giurisprudenziale de officio praesidis/proconsulis o con un liber mandatorum (quest'ultima ipotesi si deve a Dario Mantovani). 
mm 74 per il margine superiore, entrambi apparentemente conservati nella loro integrità. Sono in lingua greca - ma testimoni della scrittura maiuscola rotonda 'giuridica' fortemente ibridata che, fra gli altri, si accompagna all'onciale BR in $\mathbf{F}^{36}$ - i frammenti di codici papiracei, sempre di contenuto giuridico, P.Ant. III $152^{37}$ e PSI XIII $1348^{38}$ (VVI sec.), i più significativi esempi di un gruppo certamente più ampio. ${ }^{39}$ Del primo dei due si possono ancora misurare i margini esterno e inferiore, entrambi di ca. mm 100, mentre il secondo conserva ancora buona parte della sezione interna di un bifoglio, per un formato complessivo di almeno mm $292 \times 362$ effettivi. ${ }^{40}$ Se messi in rapporto con i quattro testimoni del Digesto, poco fa esaminati, e con i due del Codex, gli ulteriori frammenti giuridici P.Vindob. L 110, P.Ant. III 152 e PSI XIII 1348, provenienti dalle diverse località egiziane dell'Arsinoite, Antinoupolis e Ossirinco, non permettono di accantonare l'ipotesi che il modello librario (formato, impaginazione, veste grafica) rappresentato da tutti i reperti, ideato forse in ambiente orientale, potesse essere conosciuto e riprodotto localmente piuttosto che costituire opzione esclusiva di un solo centro di produzione, donde tutti gli esemplari corrispondenti fossero sistematicamente esportati. ${ }^{41}$

36 Un tentativo di definizione di questa tipologia grafica in Fressura 2016.

37 LDAB 6136; TM 64897; MP3 2979.1; CLA Suppl. 1711. Dello spazio scrittorio restano solo le estremità di alcune righe, ma Seidl 1967, 546-547, deducendo il tema della trattazione (le retentiones dotali) dai due marginalia presenti sul papiro (uno per pagina), ha ripubblicato il testo ricreando ex nibilo, sul recto, una parafrasi greca della costituzione giustinianea C. 5.13 .1 (in particolare, $\mathbb{\$}$ 5), dell'a. 530, suggerendo così una datazione del reperto agli anni seguenti la codificazione. Convalidano questa interpretazione Amelotti-Migliardi Zingale 1985, $32-34 \mathrm{n}^{\circ}$ 4, senza progredire significativamente nell'edizione del testo primario e dei due marginalia, che, in seguito, anche McNamee 2007, 507-508, non è riuscita pienamente a intendere. La rilettura di tali marginalia, in particolare, effettuata nell'ambito del progetto Redhis, ha rianimato l'ipotesi che il frammento si collochi nel dominio del diritto pregiustinianeo.

38 LDAB 5796; TM 64566; MP3 2982. Vd. almeno Ammirati 2015, 97. Riproduzione in PSIonline, <http://www.psi-online.it>.

39 Fressura 2016.

40 Tali misure, rilevate sull'originale, sono riportate da Ammirati 2015, 97.

41 E.A. Lowe propende per collocare a Costantinopoli l'origine di P.Heid. 


\section{EDIZIONE}

Testo di P.Berol. inv. 14081 stabilito tramite autopsia (maggio 2015).

\section{Trascrizione}

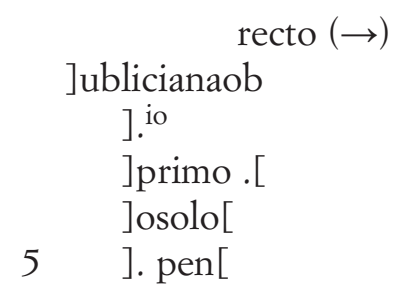

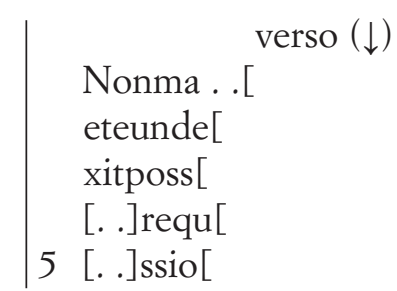

\section{Edizione $^{42}$}

inv. L 4 (CLA VIII 1221), P.Ryl. III 479 (Suppl. 1723), P.Vindob. L 110 (X 1538) e P.Ant. III 152 (Suppl. 1711), mentre assegna genericamente all'Oriente P.Pommersf. L 1-6 (IX 1351) (vd. anche, supra, nt. 26), ma la concezione dell'onciale BR - con la maiuscola rotonda 'giuridica' sua corrispondente in ambito greco - quale indizio dell'allestimento costantinopolitano di un manoscritto è stata superata a partire da Cavallo 1978, 234. La presenza di marginalia greci in P.Heid. inv. L 4 e P.Sorb. inv. 2219 (per questo vd. però quanto osservato supra, nt. 24) è parsa sufficiente a Steinwenter 1952, 134-135, per presupporre che gli esemplari originari, donde gli attuali frammenti del Digesto, non si trovassero in Egitto per un uso ufficiale, ma vi fossero giunti al seguito di individui che, conclusi gli studi di diritto a Costantinopoli o Berito, sarebbero tornati in patria portando con sé i libri di testo acquistati in quelle città; analogamente, Seidl 1954, 254.

42 Restituzioni testuali secondo Mommsen 1870. 


\section{f. recto}

[sessoris: quod et in P] ubliciana ob-

[seruatur. Superficiar [.]. \'io',

[Paulus libro uicensimo] primo ạ[d] $\quad$ D. 6.1.74 Paul. 21 ad ed.

[edictum. id est qui in alien]o solo [su-]

5 [perficiem ita habeat, ut certa]ṃ pen-

[sionem praestet,]

[Vlpianus libro sexto decimo ad]

D. 6.1.75 Ulp. 16 ad ed.

[edictum. praetor causa cognita]

[in rem actionem pollicetur.]

[Gaius libro septimo ad edictum pro-]

[uinciale. Quae de tota re uindican-]

[da dicta sunt, eadem et de parte in-]

[tellegenda sunt, officioque iudi-]

[cis continetur pro modo partis ea]

[quoque restitui iubere, quae simul]

[cum ipsa parte restitui debent. In-]

[certae partis uindicatio datur, si]

[iusta causa interueniat. Iusta au-]

[tem causa esse potest, si forte legi]

[Falcidiae locus sit in testamento,]

[propter incertam detractionem]

[ex legatis, quae uix apud iudicem]

[examinatur: iustam enim habet igno-]

[rantiam legatarius, cui homo lega-]

[tus est, quotam partem uindicare]

[debeat: itaque talis dabitur actio.]

[Eadem et de ceteris rebus intelle-]

[gemus.]

[Vlpianus libro septimo decimo ad]

[edictum Quaedam mulier fundum]

D. 6.1.76 Gai. 7 ad ed. prouinc.

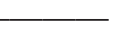

\section{f. verso}

non marị[to donauit per epistulam]

et eunde[m fundum ab eo condu-]

xit: poss[e defendi in rem ei compe-]

[te]re, qu[asi per ipsam adquisierit pos-]

5 [se]ssio[nem ueluti per colonam. pro-]

[ponebatur - - - 
recto

1 Stimata la capienza della riga (28 lettere) e osservata la posizione della parte terminale di questa in relazione con $\mathrm{r} .3$ recto, si può ritenere che la porzione iniziale pos di possessoris fosse collocata in fondo all'ultima riga della pagina precedente il fr., ora perduta. Il testo a partire all'incipit di D. 6.1.73 è restituito e.g., con una disposizione ipotizzata in base alla stima della larghezza originaria dello spazio scrittorio e alla capienza media delle righe.

2 A fine riga, lettera verosimilmente frutto di un errore dello scriba e non più distinguibile, poiché annerita, probabilmente, dal correttore. Data l'inserzione interlineare delle due lettere io, operata da quest'ultimo, è possibile che anche la precedente lettera di prima mano, ora in lacuna, risultasse errata. Non si rilevano segni di punteggiatura in chiusura di riga che corrisponde a finale di caput (punto medio, invece, nel corrispondente luogo in $\mathbf{F}, \mathrm{I}$ f. $117^{\mathrm{r}}$ ).

3 Dopo $o$, resta traccia puntiforme riconducibile all'estremità inferiore dell'occhiello di $a$; di seguito, cade interamente l'attesa $d$, oltre la quale resta supporto non scritto.

4 Dato l'allineamento verticale delle $o$ in desinenza di solo e primo di r. 3 recto, è possibile ipotizzare la presenza a fine riga della porzione iniziale su di superficiem, che per il resto doveva collocarsi a r. 5 recto.

5 Si scorge parte dell'ansa destra di $m$. Le righe seguenti sono restituite e.g. come al di sopra di r. 1 recto. Nell'inscriptio di D. 6.1.75 si restituisce e.g. sexto secondo $\mathbf{F}$, dove però Lenel 1889, 515 (Ulpianus fr. 583), propone la correzione in septimo (vd., più avanti, $\mathbb{S}$ ). Per D. 6.1.77, $\mathrm{F}$ attesta la sequenza fundum ita non, dove ita di prima mano è stato eliminato da un correttore (Korrektor 1 di Kaiser 2001, 179-183) e non accolto dal concorde Mommsen 1870, 211; il testo ricostruito per il recto del foglio cui il pap. apparteneva, al netto dell'inscriptio, doveva cominciare sull'ultima riga, ma non è possibile stimare se in esso fosse contemplata o meno la dubbia lezione ita, poiché, presente o meno, il numero di lettere per la riga di pertinenza rimarrebbe plausibile (26 contro 29 lettere).

verso

1 Resta la parte superiore di $r$ (gran parte dell'asta e dell'occhiello, ma, al di sotto, non il tratto mediano sporgente che distingue la lettera da $p$ ), seguita da traccia puntiforme dell'estremità superiore dell'attesa $i$.

3 La sporgenza a destra del tratto orizzontale di $t$ è di misura minima (cf. r. 2 verso); la sporgenza verso sinistra, anch'essa di breve estensione, si scorge a mala pena poiché quasi interamente obliterata da un danno sulla superificie scritta.

5 La lezione colonam è restituita e.g. secondo il testo di Mommsen 1870, 211 , che accoglie la lezione di prima mano in $\mathbf{F}$ (vd. app., 36) nonostante la modifica in colonum operata, a quanto pare, dal medesimo correttore che elimina ita in D. 6.1.77 (vd. r. 5 recto). Nel pap., è probabile che la riga si concludesse con le lettere pro iniziali della parola proponebatur seguente colonam. 


\section{ASPETTI DI CONTENUTO}

In P.Berol. inv. 14081 è rappresentata una porzione del titolo D. 6.1 De rei uindicatione, prossima alla sua conclusione (che avviene al fr. 80). In questo tratto finale, compiutasi la sequenza delle tre masse individuate da F. Bluhme (Edittale, fr. 1-50; Sabiniana, fr. 51-61; Papinianea, fr. 62-67) si trovano le cosiddette code, cioè frammenti fuori ordine provenienti in questo caso dalle masse Sabiniana ed Edittale (fr. 68-77). Chiudono il titolo D. 6.1 tre frammenti della cd. Appendix, secondo la regola per cui i frammenti di questo gruppo fino al titolo D. 22.3 seguono le code (mentre le precedono da D. 33.1): ciò dipende dal fatto che i frammenti dell'Appendix furono inseriti in una fase redazionale progredita, quando i titoli fino a D. 22.3 erano già stati formati. Questo fenomeno è il sintomo della rapidità con cui hanno operato i compilatori, che hanno iniziato la composizione dei titoli mentre ancora era in corso la lettura di una (piccola) parte delle opere da spogliare (lettura che era invece terminata quando la redazione arrivò al libro 33). ${ }^{43}$

Nella coda di frammenti tratti dalla massa Sabiniana e Edittale presente in D. 6.1 e parzialmente rappresentata nel papiro, i compilatori hanno operato alcuni allacciamenti testuali fra i frammenti, formando delle catene, operazione non rarissima, ma che non rappresenta neppure la regola, poiché l'ordine dei frammenti all'interno dei titoli del Digesto riflette, nella maggior parte dei casi, il puro ordine di lettura; ciò fa sì che, di solito, ogni frammento costituisca un testo a sé stante. D. 6.1.69-71 compongono una catena dedicata alle sanzioni processuali in cui incorre il convenuto con una rivendica, che abbia dismesso dolosamente il possesso. I frr. $73-75$, che cadono nella porzione di testo superstite nel papiro, si annodano a formare un'altra catena, dapprima vertente sull'onere imposto all'attore di stabilire l'eventuale quota di comproprietà sulla cosa in capo al possessore (che non è invece tenuto a dichiararla), poi sulla legittimazione attiva del superficiario, cui il pretore può concedere, valutate le circostanze, un'azione reale utile a tutela del suo diritto sulla superficie (che i Romani non avevano ancora pie-

43 Mantovani 1987, 109-124; Mantovani 2007. 
namente inquadrato nei diritti reali). Questa catena è anomala perché il frammento iniziale (73) è iscritto Ulpianus libro septimo decimo ad edictum, mentre quello conclusivo (75) è iscritto Ulpianus libro sexto decimo ad edictum (il frammento inframmezzato è di Paulus libro uicesimo primo ad edictum). Il Lenel aveva perciò proposto la più che ragionevole correzione dell'inscriptio finale in septimo decimo. ${ }^{44} \mathrm{Il}$ papiro si interrompe a r. 5 recto, senza dare la soddisfazione di una diretta conferma della proposta leneliana.

Sempre dal libro diciassettesimo ad edictum di Ulpiano proviene il fr. 77 , di cui il papiro conserva la prima parte, relativa al caso di una donna che aveva donato un fondo (non al marito: le donazioni fra coniugi erano invalide) mediante una comunicazione epistolare, contemporaneamente prendendo in locazione il fondo stesso dal donatario. La questione era se si potesse considerare trasmesso il possesso del fondo, da cui dipendeva anche l'acquisto da parte del donatario di una situazione di appartenenza tutelabile con un'azione reale (probabilmente si trattava di un fondo provinciale, sottoposto a tributo o a stipendio, per il cui acquisto era sufficiente la traditio e che legittimava ad un'azione pretoria prevista dall'editto: EP 71). ${ }^{45}$ Ulpiano risponde affermativamente: il donatario acquista l'azione reale. Lo considera insomma un caso di cd. constitutum possessorium, che si verifica quando l'attuale possessore si trasforma in detentore (a nome di altri), secondo il principio enunciato da Cels. 23 dig. D. 41.2.18 Quod meo nomine possideo, possum alieno nomine possidere: nec enim muto mibi causam possessionis, sed desino possidere et alium possessorem ministerio meo facio.

\section{Copie Del Digesto di ETÀ GIUSTINIANEA}

Con l'individuazione di questo nuovo esemplare, salgono a sei i testimoni diretti del Digesto, sicuramente identificati e risalenti all'età giustinianea o comunque al VI sec., alcuni già richiamati in precedenza:

44 Lenel 1889, 515 (Ulpianus fr. 583).

45 La fattispecie ha punti di contatto con la tematica affrontata nella costituzione di Diocleziano riferita in Vat. fr. 293; cf. Lenel, Palingenesia 1889, 515 nt. 7. 
1) il 'Codex Florentinus' (F), copia pressoché completa dei 50 libri: codice pergamenaceo su doppia colonna, in onciale BR; di origine con ogni probabilità orientale, è il prodotto di un centro scrittorio di notevole importanza, forse Costantinopoli; ${ }^{46}$

2) P.Heid. inv. L 4 (D. 5.2.17-19), ${ }^{47}$ di provenienza e origine orientale;

3) il presente P.Berol. inv. 14081 (D. 6.1.73-74, 77);

4) P.Ryl. III $479+480$ (D. $28.8 .11,30.11-13,19,22-26),{ }^{48}$ di provenienza e origine orientale;

5) P.Pommersf. L 1-6 (D. 45.1.35-36, 38, 41-46, 48-49, 72-73), ${ }^{49} \mathrm{di}$ probabile provenienza italiana e possibile origine orientale;

6) Napoli, Biblioteca Nazionale 'Vittorio Emanuele III', IV A 8, scriptio inf. ff. 36-39 (D. 10.2.3-16, 23-29; 10.4.12-19), di origine probabilmente italiana (VI sec.), costituito da due bifogli pergamenacei, con testo distribuito su un'unica colonna per pagina, in onciale non tipizzata ${ }^{50}$ i numerali latini apposti ai frammenti lo distinguono dalle copie papiracee, con numerazione greca (P. Ryl. III $479+480$, P.Pommersf. L 1-6).

7) P.Sorb. inv. 2219, codice papiraceo frammentario in scrittura minuscola ogivale inclinata, di provenienza e origine orientale, identificato con D. 19.2.54, 56?, corredato da un commento marginale attribuito a Stefano. ${ }^{51}$

Oltre ai testimoni diretti di VI secolo, si devono considerare tre manoscritti altomedievali, in quanto potrebbero fare capo ad antigrafi diretti di età giustinianea:

a) Berlin, Staatsbibliothek-Preußischer Kulturbesitz, Lat. fol. 269, ff. $183^{\mathrm{v}}-190^{\mathrm{v}}$ (D. 1.1-1.5.24 inscr.; 1.6.8 patris nascetur-1.7.3 uel praeses filius) $:^{52}$ si tratta di un quaternione contenente la fine delle

46 Vd., supra, nt. 11; sull'origine probabilmente costantinopolitana, vd. Baldi 2010, 101-102, Ammirati 2015, 96.

47 Vd., supra, nt. 16.

48 Vd., supra, nt. 17.

49 Vd., supra, nt. 18.

50 Vd., supra, nt. 24. Dopo Gaupp 1823, 'apografo' e annotazioni in Mommsen 1870, XXXX-XXXXI, LXXXX-LXXXXI, 1*-10*. Vd. anche Stolte 1999a, 115-119, Ammirati, 2015, 103.

51 Vd., supra, nt. 24.

52 Kaiser 2004, 387-415; Loschiavo 2014; Loschiavo 2015. 
Institutiones giustinianee e l'inizio del Digesto, rilegato per errore in un manoscritto contenente fra l'altro l'Epitome Iuliani e la Collatio legum Mosaicarum et Romanarum (Lex Dei). Si ritiene che il codice al quale apparteneva il quaternione sfuso (e che doveva contenere le intere Institutiones, e almeno una parte del Digesto) sia stato scritto all'inizio del IX secolo in Borgogna, forse nell'Abbazia di Flavigny-sur-Ozerain, presso Digione. L'incidente di rilegatura presuppone che si siano trovati in una stessa biblioteca il manoscritto da cui proveniva il quaternione e quello dell'Epitome Iuliani e della Lex Dei: questa compresenza implica uno spiccato interesse per le fonti giuridiche romane in Borgogna fra VIII e IX sec. Per quanto ci riguarda, il frammento del Digesto nel ms. Lat. fol. 269 è rilevante perché, secondo l'ipotesi di W. Kaiser, il quaternione corrispondente potrebbe essere stato copiato da un antigrafo in onciale BR, di origine orientale e risalente al VI sec., forse pergamenaceo. ${ }^{53}$ In questo senso, il ms. Lat. fol. 269 sarebbe seppure indiretta testimonianza di un esemplare orientale del Digesto di età giustinianea, ${ }^{54}$

b) Città del Vaticano, Biblioteca Apostolica Vaticana, Pal. Lat. 1564, ff. 124r.17-126'.22 (D. 10.1 rubr.; 1-3; 4 pr.-10; 7; 9-10; 13; 4.9-11; $6 ; 8 ; 11-12 ; 13)$ : tramanda una porzione del titolo de finium regundorum, inserita in un codice miscellaneo di argomento gromatico (collocazione che ha spinto a riorganizzare tematicamente l'ordine dei frammenti rispetto a quello originario del Digesto, come al solito meccanico). Il manoscritto è datato al IX sec.; per il Digesto,

53 La ricostruzione è basata sulla dimensione della lacuna fra D. 1.5.24 e 1.6.8, compatibile con la perdita di un foglio su due colonne (tipicamente su pergamena), di ca. 28 lettere per riga e ca. 42 righe per colonna, o due fogli su una colonna; la preferenza per l'esemplare su pergamena dipende dell'uso dell'inchiostro rosso per i nomi dei giuristi nel supposto apografo Lat. fol. 269, poiché nessuno degli esemplari del Digesto su papiro usa la rubricatura. L'antigrafo orientale fu poi glossato in Occidente (e due glosse incorporate inavvertitamente nell'apografo): Kaiser 2004, 400$405,412-414$.

54 La sua connessione con le Institutiones ha fatto proporre a Macino 2008, 25 26, l'ipotesi suggestiva che si trattasse di un manoscritto approntato ad uso scolastico, tenendo conto che nel primo anno del piano di studi giustinianeo era prevista la lettura del manuale e dei primi cinque libri del Digesto. 
il testo risale a un esemplare di età giustinianea, in alcune lezioni superiore a $\mathbf{F} .^{55}$

c) Wien, Österreichische Nationalbibliothek, Cod. 2160, f. $162^{\mathrm{va}} .30$-vb $^{\mathrm{vb}}$ (D. 22.5.12), codice di IX sec., che contiene fra l'altro l'Epitome Iuliani e la Collatio. Potrebbe avere tratto il singolo passo del Digesto da un manoscritto presente in Italia, nel luogo e tempo della probabile origine del codice miscellaneo. ${ }^{56}$

Se queste sono le testimonianze (dirette o indirette) di copie del Digesto in età giustinianea, altre tracce della circolazione orientale dell'opera, nella stessa epoca, sono costituite da PSI I 55, di provenienza ossirinchita, bifoglio di codice papiraceo di grandi dimensioni, che contiene un indice in greco di parte del titolo $2.14,{ }^{57}$ e PSI XIII 1350, quattro frammenti di un codice di papiro contenente una summa in greco (D. 2.8.12-14; 2.9.1 pr.-1; 2.11.2.8-9; 2.11 .4 pr., 1, 5). ${ }^{58}$ A queste testimonianze va aggiunta ovviamente la fiorente attività di traduzione, riassunto e commento dei maestri, in parte confluita nei più tardi Basilici..$^{59}$

Eco molto flebile del Digesto è rimasta nella letteratura extra-giuridica contemporanea, praticamente solo in Giovanni Lido. ${ }^{60}$

55 Riproduzione in Digital Vatican Library, <http://digi.vatlib.it/view/MSS_Pal. lat.1564>. Lachmann 1848; Mommsen 1870, XXXXI, 306-308 (apparato); Thulin 1913; Stolte 1992; Kaiser 2013, 273-347. Due brevi estratti, forse ricavati dal testo più ampio, si trovano nello stesso Pal. lat. 1564, f. $3^{\mathrm{r}} \cdot 11-16$ (D. 10.1.4.1, 4). A differenza di quanto riteneva Mommsen, è da considerare un apografo del manoscritto vaticano il Wolfenbüttel, Herzog August Bibliothek, Gud. lat. 105, ff. 81 ${ }^{\mathrm{r}}-83^{\mathrm{r}}$.

56 Riproduzione in Österreichische Nationalbibliothek, <http://data.onb.ac.at/ rec/AL00172917>. Kaiser 2004, 153-165; Kaiser 2013, 343-344. In questo caso, a differenza dei precedenti, la datazione dell'antigrafo all'età giustinianea è indimostrabile.

57 LDAB 2553; TM 61409; MP3 2965. Brandsma 1996, 113-118; Falcone 2001; Ammirati 2015, 99. Riproduzione in PSIonline, <http://www.psi-online.it >.

58 LDAB 2552; TM 61408; MP33 2279. La Pira 1930; van der Wal 1983, 31 nt. 5 , 33 nt. 15; Brandsma 1996, 105-112; Ammirati 2015, 99. Riproduzione in PSIonline, $<$ http://www.psi-online.it>.

59 Per tutti, Stolte 1999a, 112-113.

60 Su Lido, vd., qui, $\$ 6$. 


\section{LA CIRCOLAZIONE DEL DigESTO: PROSPETTIVE DI METODO}

Costituisce un problema storico di notevole importanza se i tria uolumina ${ }^{61}$ fatti comporre da Giustiniano nonché la legislazione che ha accompagnato e seguito l'attività compilatoria abbiano trovato effettiva applicazione nell'esperienza giuridica coeva. Rispetto alle risposte negative, da V. Arangio-Ruiz fino alla radicale e insostenibile negazione di A. Schiller, ${ }^{62}$ di recente - riattivando un'impostazione già abbozzata da A. Steinwenter ${ }^{63}$ - si sta imponendo una visione più equilibrata, sollecitata dalla raccolta di M. Amelotti e L. Migliardi Zingale e consolidata dai ripetuti interventi di J. Beaucamp. ${ }^{64}$ Se Schiller nel 1970 poteva affermare che «[the] contemporaneous legislation emanating from Constantinople likewise had no impact on the current law in Egypt» ed era «practically unknown and almost totally disregarded in practice», ${ }^{65}$ questi nuovi studi hanno registrato con pazienza i riflessi della legislazione giustinianea (specialmente novellare) nella prassi egiziana (che resta l'osservatorio pressoché obbligato per ragioni documentali), concludendo che «Justinian's legislation, including the Novels, was thus known to the elites of the empire or rather to the jurists whom they employed».66

Da questa revisione, tuttavia, è rimasto di fatto escluso il Digesto. Mentre la crescente attenzione per la documentazione papirologica ha portato a rivedere l'opinione che negava alla legislazione giustinianea un impatto effettivo nell'esperienza giuridica contemporanea, sul Digesto continua a gravare l'idea suggerita - forse involontariamente da G. Rotondi nel 1918, quando concludeva che se dovessimo fare affidamento solo sulle fonti letterarie, «sul Digesto e sulle Istituzioni non

61 Il termine uolumen, qui e altrove adoperato in conformità al lessico giustinianeo con riferimento alle tre opere che compongono il Corpus iuris, non dev'essere confuso con il termine tecnico bibliologico che identifica il formato rotolo.

62 Arangio-Ruiz 1920; Schiller 1970; Schiller 1971.

63 Steinwenter 1952; più restrittivo circa la penetrazione delle Novelle nella prassi egiziana, Steinwenter 1958, 34; cf. Taubenschlag 1940-1941.

64 Amelotti-Migliardi Zingale 198; Beaucamp 2005; Beaucamp 2007; Keenan 2014; cf. anche Pieler 2000.

65 Schiller 1970, 45.

66 Beaucamp 2007, 281. 
avremmo nessunissima notizia, e così la parte veramente più notevole del lavoro di compilazione ci sfuggirebbe completamente». ${ }^{67}$ Questa constatazione si è trasformata in un giudizio complessivo, fino a fare pensare «que la littérature sur le Digeste servait seulement au besoin théorique de conserver en mémoire une partie de la connaissance scolaire». ${ }^{68}$

$\mathrm{Su}$ questo risultato pesano vari fattori che meritano di essere almeno accennati, di carattere storiografico e relativi alla tipologia delle fonti disponibili. Sul piano storiografico, ha esercitato un forte condizionamento sulla ricerca l'orientamento prevalente nella prima metà del $\mathrm{XX}$ sec. e anche oltre, che attribuisce l'iniziativa di comporre il Digesto ad una tendenza non solo classicista, ma addirittura arcaistica di Giustiniano: un gusto culturale elitario e non condiviso, che spiegherebbe la fredda accoglienza riservata dai contemporanei a questa parte del Corpus iuris. ${ }^{69}$ L'abbandono delle opere dei giuristi classici in età pre-giustinianea, che spiegherebbe l'insuccesso del loro recupero tentato dal Digesto, è tuttavia un assunto indimostrato.

Ma è soprattutto la tipologia delle fonti disponibili il fattore che può introdurre le maggiori distorsioni, quando si indaghi la maggiore o minore influenza delle varie parti della compilazione giustinianea e della legislazione novellare. ${ }^{70}$ Gli autori non-giuristi (per es. storici, cronachisti, tecnigrafi) hanno in generale poche ragioni di citare le fonti giuridiche (che è la constatazione fatta da Rotondi per l'insieme del Corpus iuris): quando lo fanno, la loro attenzione si appunta su

67 Rotondi 1918, 263-264, che non si riferisce peraltro a una presunta ignoranza dell'esistenza del Digesto da parte delle fonti letterarie, ma al fatto che tali fonti non sembrino dare alcun rilievo particolare all'impresa legislativa giustinanea in quanto tale (si limitano semmai a citarlo sporadicamente per i contenuti). Considerato che il nostro interesse si orienta soprattutto sulla tradizione manoscritta del Digesto, di questi sparsi testimonia indiretti - fra i quali si annovera la nota epistola di Gregorio Magno del 603 (reg. ep. 13, 49 [50]) - non dobbiamo qui occuparci, rimandando a Mommsen 1870, XXXXII, Stolte 1999a, 112, Kaiser 2008.

68 Beaucamp 2005, 28, pur in un contesto molto prudente ed equilibrato.

69 Per una rivisitazione concettuale, Puliatti 2013.

70 Riflessioni importanti sulla distorsione delle fonti nella ricostruzione del diritto bizantino, anche se in prospettiva diversa dalla presente, in Simon 1988, Stolte 1998 e Beaucamp 2005, 5-8, con accento sulla differenza fra 'Law in books' e 'Law in action'. 
innovazioni o comunque su disposizioni rilevanti sul piano socio-politico, cioè su costituzioni imperiali. È ben difficile viceversa che abbiano motivo di citare brani giurisprudenziali quali quelli contenuti nel Digesto.

Questo principio può essere verificato, per es., esaminando le citazioni contenute nella Chronographia di Giovanni Malala. Nel logos XVIII dedicato al regno di Giustiniano, lo storico siro mette innanzitutto in rilievo le leggi emanate dallo stesso imperatore: $:^{71}$ una costituzione sui limiti imposti ai magistrati, nelle loro province, nella costruzione di case o negli acquisti fondiari (disposizione identificabile con C. 1.53.1 del 528), di cui Malala mette in rilievo l'intento di protezione verso i sudditi; una costituzione che concedeva la successione ai figli naturali (probabilmente C. 5.27.8-9 del 528); una costituzione che accordava all'erede la facoltà di accettare senza limiti di tempo (C. 6.30.19 del 529 o C. 6.30 .22 del 531), entrambe citate come esempio dell'attenzione alla trasmissione dei patrimoni; una costituzione sull'obbligo di testimoniare (verosimilmente C. 4.20.16, forse del 527). ${ }^{72}$ Sempre con l'intento di illustrare l'azione di governo di Giustiniano nel suo complesso, Malala, a proposito dell'anno 528, accenna comprensibilmente al Codex in quanto raccolta di nomoi di imperatori precedenti e dello stesso Giustiniano, e tace altrettanto comprensibilmente, in questa prospettiva, del Digesto e delle Institutiones. ${ }^{73}$ Il silenzio su queste ultime due parti è del resto ancora più comprensibile se, come si ritiene generalmente, anche se con varie sfumature, la Chronographia fu redatta in due fasi, la prima delle quali si arrestereb-

71 Si tiene presente il testo di Thurn 2000, dove si trovano sistematiche indicazioni di concordanza con l'edizione Dindorf 1831.

72 Malal. 18.20. Per le identificazioni, Rotondi 1918, 243-244, e, indipendentemente da costui, Corcoran 2008, 86-88; sul contributo di Rotondi si basa invece l'importante rivisitazione di Scott 2012, che per la costituzione relativa alla successione dei figli naturali preferisce l'identificazione con C. 4.57.5, pur notando vari problemi di contenuto e di datazione, poiché il testo è successivo alla prima edizione del Codex.

73 Malal. 18.20; la notizia è ripetuta per l'anno 529 in 18.38, in cui si aggiunge che lo scopo era di evitare svantaggi ai litiganti e di accelerare i processi; il $\mu$ ovó $\beta ı \beta \lambda o v$ fu spedito ad Atene e Berito. Sulla duplicazione, Scott 2012, 20. 
be al 532, dunque prima della pubblicazione del Digesto e delle Institutiones, mentre la parte posteriore riporta gli eventi del regno di Giustiniano in modo assai più stringato. ${ }^{74}$ Anche quando, nel corso della narrazione, Malala menziona singoli provvedimenti, non stupisce che dia «molto maggiore rilievo alle singole disposizioni nuove»come notava già Rotondi ${ }^{75}$ - e che la scelta dipenda dalla connessione con avvenimenti narrati dallo storico e con la prospettiva apologetica della sua cronaca. Ciò vale per le riforme nell'amministrazione dell'Armenia e per la norma (identificabile con C. 1.3.42) che, per evitare fenomeni di malversazione, faceva divieto ai vescovi o amministratori di opere pie di disporre per testamento del patrimonio acquistato dopo l'assunzione dell'incarico. ${ }^{76}$ Lo stesso si applica alle altre norme sporadicamente citate, scelte perché toccavano materie sensibili dal punto di vista sociale o politico: la repressione dell'omosessualità, provocata da uno scandalo in cui erano stati coinvolti alcuni vescovi (Malal. 18.18); il divieto per i pagani di assumere cariche pubbliche, sancito da un editto che Malala richiama nel contesto di un episodio di persecuzione del paganesimo (18.42); la chiusura della scuola di filosofia neoplatonica e - meno verosimilmente - di diritto di Atene (rilevante nella prospettiva della cristianizzazione del mondo che anima la Chronographia) e il divieto del gioco dei dadi, posto in relazione con alcuni episodi con risvolti di blasfemia, duramente repressi (18.47); ${ }^{77}$ la limitazione delle spese giudiziarie e delle sportule (forse Nov. 82.7 del 539), con esplicito riflesso sociale (18.67; cf. 18.38); la difesa dell'ortodossia, sancita dall'edictum de orthodoxa fide, aduersus impia baereticorum dogmata (18.78), ricordato per il suo significato religioso, così come la pronuncia contro i Monofisiti (18.142). Nella scelta dei prov-

74 La prima parte si estende verosimilmente fino al cap. 18.75 (oppure 78); sulla datazione della sua stesura - compiuta verosimilmente ad Antiochia - i commentatori sono più incerti, oscillando fra 528 e 550: per tutti, Puech 2006. Dopo il cap. 78, per il periodo fino alla fine del regno di Giustiniano, è citata una sola norma (18.142: editto religioso contro i Monofisiti).

75 Rotondi 1918, 346.

76 Malal. 18.10.

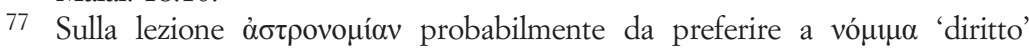
(accolta nell'ed. Dindorf 1831), Watts 2004; cf. Corcoran 2008, 102-103. 
vedimenti citati, oltre il rilievo storico e l'intento apologetico, influisce anche il fatto che taluni di essi erano stati inviati ad Antiochia, la città d'origine di Malala (come l'edictum sulle spese giudiziarie, scritto in greco, che era stato incluso nell'archivio pubblico della città) o più in generale esposti in luoghi dove lo storico siro poteva averne avuta conoscenza. ${ }^{78}$ Come è stato ben scritto, «the codification might well have seemed a fine achievement to Justinian, but it apparently failed to capture the imagination of the ordinary Greek-speaking citizen, for whom the action of simply collecting and editing old laws in Latin probably seemed both remote and irrelevant. But these new laws, at least as described by Malalas, provided on the other hand solid practical evidence of the value of Justinian as legislator»». ${ }^{79} \mathrm{Vi}$ è dunque una ragione se la Chronographia privilegia le costituzioni di Giustiniano ed è altrettanto comprensibile il silenzio sul Digesto, senza che il diverso trattamento riservato da Malala nella sua storia del mondo in prospettiva cristiana sia un indice del diverso valore che le due collezioni avevano sul piano giuridico e dell'effettività.

Un altro esempio può fornire una controprova. Il Digesto è citato ripetutamente nel de magistratibus populi Romani di Giovanni Lido, ma anche queste citazioni confermano che l'interesse degli scrittori non giuristi non riguardava il diritto privato. ${ }^{80}$ Traendoli dal Digesto, Lido cita Pomponio l.s. enchirid. D. 1.2.2.22-23 = Lyd. 1.26.2-3, sull'origine regia della questura e sui quaestores parricidii; D. 1.2.2.3-4 = Lyd. 1.34.1, sul decemvirato; D. 1.2.2.34 = Lyd. 1.48.3-4, sui magistrati giusdicenti; Ulpiano de off. quaest. D. 1.13.1 = Lyd. 1.24.6, sull'origine e le competenze dei questori; D. 1.13.1-2 = Lyd. 1.28.1, sui quaestores candidati Caesaris; Ulpiano de omn. trib. $=$ Lyd. 1.48.5, sui pretori tutelari e fedecommissari (la citazione è però un rinvio all'opera); Paolo de off.

78 Di invio di una costituzione in varie città dell'impero Malala parla a 18.38, 42; di esposizione in varie chiese si parla in 18.142. Antiochia è il punto di vista da cui si guarda agli avvenimenti nella prima parte dell'opera, fino al 532: cf. Thurn 2000, 3*, Croke 1990.

79 Scott 2012, 14.

80 Sui giuristi come fonte di Lido, Dubuisson-Schamp 2006, CLXXXIIICXCVII, anche per la confusione fra Gaio e Pomponio. I brani sono citati secondo questa edizione. 
praef. uig. D. 1.15.1 = Lyd. 1.50.6, sui tresuiri capitales; Arcadio Carisio de off. praef. pr. D. 1.11.1 = Lyd. 1.14.2-5. Il Digesto è evidentemente sfruttato da Lido esclusivamente per quanto ha da offrirgli per la storia delle magistrature romane: il diritto privato, ancora una volta, si dimostra fuori dell'orizzonte d'interesse degli scrittori non giuristi. Se si tiene in giusto conto l'intento di queste opere, si vede che sarebbe indebito trarne la conclusione che il Digesto sia stato estraneo anche alle scuole di diritto, ai giudizi e alla prassi negoziale. Proprio il fatto che un autore non interessato al diritto privato, come Lido, abbia utilizzato il Digesto è semmai un segno della sua circolazione.

Anche la documentazione della prassi dev'essere esaminata nella giusta luce: indubbiamente essa consente di avvicinarsi al piano della concreta applicazione del diritto, ma a anche in questo tipo di documenti (come testamenti e contratti) non c'era frequentemente motivo di citare brani del Digesto. Come ha giustamente rilevato Beaucamp, «le droit officiel et la législation impériale sont particulièrement pris en compte en Égypte dans leur aspects publics, procéduraux ou formels». ${ }^{81}$ Non stupisce perciò che le citazioni più sicure - anche se talune non identificabili con certezza - si riferiscano a Novelle. ${ }^{82}$ Per il diritto privato l'influenza del diritto ufficiale la si deve cercare semmai ad un livello più intrinseco: ad esempio, si coglie nel lessico ed è certamente da differenziare secondo il livello sociale. ${ }^{83}$

Sia la letteratura extra-giuridica sia i documenti della prassi, per quanto rilevantissimi sotto altri aspetti, non offrono dunque un osservatorio adatto per valutare l'impatto del Digesto. Già G. Lanata aveva notato che le testimonianze dirette dei libri giustinianei, compreso il Digesto, sono invece un indice prezioso, non ancora adeguatamente valorizzato. ${ }^{84}$ La pubblicazione di un frammento di una nuova copia del Digesto, di età giustinianea, suggerisce di porre di nuovo il problema. Bastino qui sommarie osservazioni.

\footnotetext{
81 Beaucamp 2005, 39.

82 Vd. per tutti Amelotti-Migliardi Zingale 1985, 89-93, Beaucamp 2005, 11-15,

83 Così ancora Beaucamp 2005, 41-43.

84 Lanata 1984, 19-24, ove già un'importante ricognizione.
} Corcoran 2007. 
Comparando le testimonianze dirette del Digesto con quelle delle Institutiones e del Codex, risulta che il Digesto è, fra i tria uolumina, il più rappresentato in età giustinianea.$^{85}$ Delle Institutiones residua il solo palinsesto Verona, Biblioteca Capitolare, XXXVIII (36), la cui scriptio inferior, in scrittura onciale, è riferita al VI-VII sec. ${ }^{86}$ Del Codex repetitae prelaectionis, ${ }^{87}$ si annoverano entro il VI sec.:

1) PSI XIII 1347 (C. 7.16.41-42; 17.1), frammenti di un foglio di codice papiraceo, con glosse greche interlineari (traduzioni di termini latini) ${ }^{88}$

2) Verona, Biblioteca Capitolare, LXII (60), scriptio inf. ff. 4-81 (C. 4.48.2-8.54; 11.61.6-12.36.4) $;{ }^{89}$

3) Köln, Historisches Archiv, GB Kasten B 130 + Münster, Universitäts- und Landesbibliothek, 718m (1186) (†1945) (C. 3.32.4-12), datato al VI secolo e di probabile origine italiana. ${ }^{90}$

Il confronto è nettamente a favore del Digesto, che da solo conta poco meno del doppio di esemplari rispetto al totale di quelli delle due altre parti. ${ }^{91}$

85 Si prescinde qui dalle ulteriori attestazioni più sopra elencate che - seppur indirettamente - rivelano la presenza di antigrafi di VI sec.: sarebbe necessario, infatti, esaminare nella medesima prospettiva anche la tradizione altomedievale del Codex Iustinianus e delle Institutiones. Si prescinde inoltre dalla testimonianza ricavabile dalle fonti scritte indirette (cf., supra, nt. 67), sulle quali vd. Conrat 1891, Conrat 1913, Rotondi 1918.

86 LDAB 7851; TM 66603; CLA IV 495. Macino 2008, 29; Ammirati 2015, 99.

87 Sono riferibili alla prima edizione del Codex: P.Oxy. XV 1814, indice (vd., supra, nt. 30); P. Sorb. inv. 2173 (LDAB 2555; TM 61411; MP33 2966.1; CLA V 700), frammento di bifoglio di codice papiraceo (VI sec.), con resti di testi compresi fra C. 12.59(60).10.5 e 12.62(63).4: vd. per tutti Amelotti-Migliardi Zingale 1985, 24-26 nº 2, Ammirati 2015, 99. Riproduzione in Institut de Papyrologie de la Sorbonne cit., $<$ http://www.papyrologie.paris-sorbonne.fr/menu1/collections/platin.htm> (vd., supra, nt. 24). Sulla storia della composizione del Codex e la sua tradizione manoscritta, Corcoran 2016.

88 Vd., supra, nt. 31.

89 LDAB 7868; TM 66620; CLA IV 513. Ammirati 2015, 95.

90 LDAB 8575; TM 67307; CLA VIII 1167. Ammirati 2015, 103.

91 Su questa statistica può incidere la maggiore mole del Digesto, che ne favorisce proporzionalmente la sopravvivenza. Per contro, la maggiore mole avrà reso più impegnativo moltiplicare le copie. 
Anche la distribuzione è significativa, poiché il Digesto è bene attestato sia in Oriente sia in Occidente. L'Oriente è probabilmente il luogo d'origine del 'Codex Florentinus' e di P.Pommersf. L 1-6, e sicuramente di tutte le altre copie papiracee, ${ }^{92}$ provenienti dall'Egitto (il luogo di copia non può essere determinato), cioè da luoghi distanti dai principali centri di cultura giuridica, Costantinopoli e Berito: segno di una buona circolazione, quali che ne fossero i percorsi e i moventi.

Quanto all'Occidente, il palinsesto napoletano IV A 8 dimostra la disponibilità di manoscritti in Italia, subito dopo l'estensione della legislazione, in età giustinianea; ed è nello stesso periodo che proprio in Italia sembrerebbero giunti anche il 'Codex Florentinus'93 e P.Pommersf. L 1-6. ${ }^{94}$ In questo senso, essi sono coerenti con la trasmissione dei testi della compilazione, testimoniata retrospettivamente da Giustiniano nella pragmatica sanctio del 554 (Epitome Iuliani, Appendix $A$ ), e con la volontà che essi trovassero effettiva applicazione: l'invio in Italia potrebbe risalire al 540 circa. ${ }^{95}$ Ovviamente l'Italia è un ambiente con sue caratteristiche specifiche, ma di per sé lo sforzo compiuto dalla corte di Costantinopoli di trapiantare il Digesto nei territori riconquistati è un sintomo della determinazione che accompagnò l'impresa codificatoria.

La tipologia libraria è suggestiva di una varietà di intenti e di usi. Il 'Codex Florentinus' - per numero dei copisti che lo hanno realizzato, perizia nel latino e nel greco, cura della forma - potrebbe costituire «una copia 'ufficiale' del Digesto, forse destinata a una magistratura di alto rango»: ${ }^{96}$ in altri termini, una copia uscita dall'ingranaggio di produzione, disseminazione e controllo previsto come elemento stesso di successo dell'impresa legislativa (Const. Tanta/ $\Delta \dot{\delta} \delta \omega \kappa \varepsilon v 24)$. Lo stesso si

92 Si ritiene orientale anche il manoscritto da cui discende il quaternione inserito nel Berlin, Staatsbibliothek-Preußischer Kulturbesitz, Lat. fol. 269, comprensivo in questo caso anche delle Institutiones.

93 Kaiser 2000, 111-114.

94 Sirks-Sijpesteijn-Worp 1996, 137-142.

95 Corpus iuris cinilis III = Schoell-Kroll 1954, 799-802 (Appendix VII); Kaiser 2010, 193-200.

96 Ammirati 2015, 96. 
potrebbe affermare, nonostante l'apparente differenza tipologica, per i quattro esemplari in onciale BR su papiro (P.Berol. inv. 14081; P.Heid. inv. L 4; P.Ryl. III 479 + 480; P.Pommersf. L 1-6), anche se la loro conservazione in ridottissimi frammenti non permette di escludere altre ipotesi, quali la circolazione sul mercato per l'acquisto a uso personale o, soprattutto, scolastico. Quest'ultima interpretazione sembra ragionevolmente compatibile con la tipologia rappresentata da P.Sorb. inv. 2219 , testimone, come già osservato, di un ampio commento marginale che potrebbe essere considerato trascrizione - per dire così - diretta dell'insegnamento di Stefano; ma anche P.Heid. inv. L 4 reca a sua volta annotazioni marginali rivelatrici di un'accurata comprensione di D. 5.2.18-19.97 Vanno aggiunti - perché provano in modo se possibile ancor più diretto la penetrazione nella prassi scolastica del Digesto, che vi viene tradotto in greco e commentato o riassunto - PSI I 55 e PSI XIII 1350.

Infine, sebbene lo stato frammentario di questi esemplari, eccezion fatta per il 'Codex Florentinus', renda difficile conclusioni certe, vi sono sintomi che fanno pensare a una circolazione di copie complete (ancorché suddivise in più tomi, come si è ipotizzato qui sopra per P.Berol. inv. 14081). Nelle copie pervenute sono rappresentati: il libro 5, della pars I (P.Heid. inv. L 4) ${ }^{98}$ il libro 6, della pars II de iudiciis (P.Berol. inv. 14081) ${ }^{99}$ il libro 19, della pars III de rebus (P.Sorb. inv. 2219); i libri 28 e 30, della pars $V$ de testamentis e de legatis et fideicommissis (P.Ryl. III $479+480$ ); il libro 45, della pars VII (P.Pommersf. L 1-6). Quest'ultima presenza spicca, perché l'uso scolastico del Digesto era limitato ai primi 36 libri, restando gli ultimi 14, delle due partes finali, riservati all'uso personale, $u t$ possint postea eos legere et in iudiciis ostendere (Const. Omnem 5).

Seppure il campione resti esiguo per trarre conclusioni definitive,

97 Vd. per ora McNamee 2007, 497 (P.Heid. inv. L 4: tutte le note sintetizzano accuratamente i passi del Digesto, compresa la 'glossa III', che non si distacca da D. 5.2.19), 499 (dove sono considerati unitariamente P.Sorb. inv. 2219 e P.Sorb. inv. 2173: in realtà solo il primo è attribuibile al Digesto; vd., supra, nt. 24), 496-497 (PSI I 55).

98 A questa pars appartiene anche il libro 1, rappresentato dal ms. Berlin, Staatsbibliothek-Preußischer Kulturbesitz, Lat. fol. 269.

99 A questa pars appartiene anche il libro 10, rappresentato dal ms. Città del Vaticano, Biblioteca Apostolica Vaticana, Pal. Lat. 1564. 
chi consideri l'evidenza papirologica della trasmissione diretta - accresciuta da P.Berol. inv. 14081 - vede stagliarsi sugli altri uolumina proprio il Digesto. Naturalmente, si potrebbe ritenere che questo accertamento non faccia che confermare l'accoglienza favorevole riservata dalle scuole. Connettere la maggiore diffusione con il solo uso scolastico sarebbe tuttavia un argomento improprio, poiché il piano di studi prevedeva anche la lettura delle Institutiones e delle constitutiones contenute nel Codex (al quinto anno: Const. Omnem 5), opere sopravvissute in minor numero di copie. Il punto fondamentale è, però, un altro: relegare la maggior parte degli esemplari del Digesto agli ambienti scolastici, e con ciò considerare la diffusione delle sue copie come irrilevante per provare la penetrazione nell'esperienza giuridica, si fonda su un'incongrua scissione fra prassi e insegnamento, fra 'Law in books' e 'Law in action': ${ }^{100}$ quasi che l'insegnamento scolastico non fosse la base più consistente per formare giuristi e giudici capaci di applicare nella prassi il medesimo diritto appreso nelle scuole.

\section{BIBLIOGRAFIA}

(Sigle dei periodici, dove possibile, secondo Centro Italiano dell'Année Philologique. Siglario, <http://www.aristarchus.unige.net/CIAPh/it-IT/Database/Siglario >)

Amelotti-Migliardi Zingale 1985, M. Amelotti - L. Migliardi Zingale, Le costituzioni giustinianee nei papiri e nelle epigrafi, Milano, A. Giuffrè, $1985^{2}$.

Ammirati 2010, S. Ammirati, Per una storia del libro latino antico. Osservazioni paleografiche, bibliologiche e codicologiche sui manoscritti latini di argomento legale dalle origini alla tarda antichità, JJP 40 (2010), 55-110.

Ammirati 2015, S. Ammirati, Sul libro latino antico. Ricerche bibliologiche e paleografiche, Pisa-Roma, F. Serra, 2015.

Arangio-Ruiz 1920, V. Arangio-Ruiz, Applicazione del diritto giustinianeo in Egitto, Aegyptus 1 (1920), 21-36 = Id., Studi epigrafici e papirologici, Napoli, Giannini, 1974, 1-13.

Baldi 2010, D. Baldi, Il Codex Florentinus del Digesto e il 'Fondo Pandette' della

$100 \mathrm{Da}$ cui non era immune persino Steinwenter 1952, 134-135, quando argomentava che i frammenti egiziani del Digesto sarebbero stati prove rilevanti dell'uso dell'opera nella prassi solo se si fosse potuto dimostrare che essi provenivano da archivi di magistrati. 
Biblioteca Laurenziana (con un'appendice di documenti inediti), S\&T 8 (2010), 99-186.

Beaucamp 2005, J. Beaucamp, L'bistoire du droit byzantin face à la papyrologie juridique. Bilan et perspectives, in L. Burgmann (hrsg.), Fontes Minores XI, Frankfurt am Main, Lowenklau, 2005, 5-55.

Beaucamp 2007, J. Beaucamp, Byzantine Egypt and imperial law, in in R.S. Bagnall (ed.), Egypt in the Byzantine World, 300-700, Cambridge, Cambridge University Press, 2007, 271-287.

Boyé 1926, A.J. Boyé, Essai critique sur une crux iuris. La loi Mater (Dig. V, 2, 19) et le Papyrus de Heidelberg 1272, TRG 5 (1926), 464-488.

Brandsma 1996, F. Brandsma, Dorotheus and his Digest translation, Groningen, E. Forsten, 1996.

Cameron - Ward-Perkins- Withby 2000, A. Cameron - B. Ward-Perkins - M. Withby, The Cambridge Ancient History XIV. Late Antiquity: Empire and Successors, A.D. 425-600, Cambridge, Cambridge University Press, 2000.

Cavallo 1978, G. Cavallo, La circolazione libraria nell'età di Giustiniano, in G.G. Archi (a cura di), L'Imperatore Giustiniano. Storia e mito (Giornate di studio a Ravenna, 14-16 ottobre 1976), Milano, A. Giuffrè, 1978, 201-236.

Cavallo 1996, G. Cavallo, Iniziali, scritture distintive, fregi. Morfologie e funzioni, in C. Scalon (a cura di), Libri e documenti d'Italia: dai Longobardi alla rinascita delle città (Atti del Convegno Nazionale dell'Associazione Italiana Paleografi e Diplomatisti, Cividale, 5-7 ottobre 1994), Udine, Arti Grafiche Friulane, 1996, 15-33.

Cavallo-Fioretti 2014, G. Cavallo - P. Fioretti, Chiaroscuro. Oltre l'angolo di scrittura (secoli I a.C.-VI d.C.), Scripta 7 (2014), 29-64.

Cherubini-Pratesi 2010, P. Cherubini - A. Pratesi, Paleografia latina. L'avventura grafica del mondo occidentale, Città del Vaticano, Scuola Vaticana di Paleografia, Diplomatica e Archivistica, 2010.

CLA, E.A. Lowe, Codices Latini antiquiores. A Palaeographical Guide to Latin Manuscripts Prior to the Ninth Century, Oxford, Clarendon Press, 1934-1971.

CLA Add. I, B. Bischoff - V. Brown, Addenda to Codices Latini Antiquiores, MS 47 (1985), 317-366.

Collinet 1925, P. Collinet, Études bistoriques sur le droit de Justinien II. Histoire de l'école de droit de Beyrouth, Paris, Recueil Sirey, 1925.

Conrat 1891, M. Conrat (Cohn), Geschichte der Quellen und Literatur des römischen Rechts im früheren Mittelalter I, Leipzig, J. C. Hinrichs, 1891.

Conrat 1913, M. Conrat (Cohn), Römisches Recht im frühesten Mittelalter, ZRG 34 (1913), 13-45.

Corbino-Santalucia 1988, A. Corbino - B. Santalucia, Justiniani Augusti pandectarum codex Florentinus, Firenze, Olschki, 1988.

Corcoran 2007, S. Corcoran, Two Tales, Two Cities: Antinoopolis and Nottingham, in J. Drinkwater - B. Salway (eds.), Wolf Liebeschuetz Reflected: Essays Presented by Colleagues, Friends, \& Pupils, London, Institute of Classical Studies, University of London, 2007, 193-203.

Corcoran 2008, S. Corcoran, Justinian and his two Codes: revisiting P. Oxy. 1814, JJP 38 (2008), 73-111. 
Corcoran 2016, S. Corcoran, The Codex of Justinian: The Life of a Text through 1,500 Years, in The Codex of Justinian. A New Annotated Translation, with Parallel Latin and Greek Text I, Cambridge, Cambridge University Press, 2016, XCVIICLXIV.

Cribiore 2007, R. Cribiore, The School of Libanius in Late Antique Antioch, PrincetonOxford, Princeton University Press, 2007.

Croke 1990, B. Croke, Malalas, the man and his work, in E. Jeffreys - B. Croke - R. Scott (eds.), Studies in Jobn Malalas, Sydney, Australian Association for Byzantine Studies, 1990, 1-25.

CPL, R. Cavenaile, Corpus papyrorum Latinarum, Wiesbaden, Harrassowitz, 1958.

Demicheli 2000, A.M. Demicheli, L'editto XIII di Giustiniano in tema di amministrazione e fiscalità dell'Egitto bizantino, Torino, G. Giappichelli, 2000.

de Ricci 1912, S. de Ricci, Deux nouveaux papyrus juridiques, in Études d'bistoire juridique offertes à Paul Frédéric Girard I, Paris, P. Geuthner, 1912, 273-282.

Dindorf 1831, L. Dindorf, Ioannis Malalae chronographia, Bonnae, Weber, 1831.

Dubuisson-Schamp 2006, M. Dubuisson - J. Schamp, Jean Le Lydien. Des magistratures de l'état romain I, 1, Paris, Les Belles Lettres, 2006.

Düll-Seidl 1941, R. Düll - E. Seidl, Ein Digestenfragment aus Ägypten, aber kein „predigesto de legatis", ZRG 61 (1941), 406-410.

Elliott 1999, J.K. Elliott, The Biblical Manuscripts of the Jobn Rylands Library of Manchester, BRL 81, 2 (1999), 3-50.

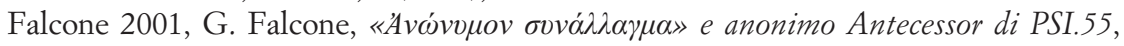
MEP 4, 6 (2001), 513-529.

Falcone 2014, G. Falcone, The probibition of commentaries to the Digest and the antecessorial literature, SGron 9 (2014), 1-36.

Fressura 2016, M. Fressura, PSI XIII 1306: note codicologiche e paleografiche, in N. Pellé (a cura di), Spazio scritto e spazio non scritto nel libro papiraceo. Esperienze a confronto (Atti della Seconda Tavola Rotonda del Centro di Studi Papirologici dell'Università del Salento, 9 ottobre 2014), Lecce, Pensa Multimedia, 2016, 77-128.

Fressura-Mantovani 2017, M. Fressura, D. Mantovani, P.Berol. inv. 14081. Frammento di una nuova copia del Digesto di età giustinianea, Athenaeum 105 (2017), 689-714.

Gaupp 1823, E.Th. Gaupp, Quatuor folia antiquissimi alicujus Digestorum codicis rescripta Neapoli nuper reperta, Vratislaviae, 1823.

Gerhard 1907, G.A. Gerhard, Zum Heidelberger Digestenpapyrus, Philologus 66 (1907), 477-480.

Gerhard-Gradenwitz 1903, G.A. Gerhard - O. Gradenwitz, Glossierte Paulusreste im Zuge der Digesten. Ein Heidelberger Papyruscodex, Philologus 62 (1903), 95-124.

Girard-Senn 1967, P.F. Girard - F. Senn, Textes de droit romain I, Paris, Dalloz, $19677^{7}$.

Gradenwitz 1902, O. Gradenwitz, Glossirte Paulusreste im Zuge der Digesten, ZRG 23 (1902), 458-459.

Herrmann 1966, J. Herrmann, Die Papyrussammlung in Pommersfelden, in Atti dell'XI Congresso Internazionale di Papirologia (Milano, 2-8 settembre 1965), Milano, Istituto Lombardo di Scienze e Lettere, 1966, 188-194 = Id., Kleine Schriften zur Rechtsgeschichte, München, C.H. Beck, 1990, 138-151. 
Irigoin 2009, J. Irigoin, Il libro greco dalle origini al Rinascimento, Firenze, Istituto Papirologico G. Vitelli, 2009.

Kaiser 2000, W. Kaiser, Zum Aufbewahrungsort des Codex Florentinus in Süditalien, in F. Theisen - W.E. Voß (hrsg.), Summa - Glosse - Kommentar. Juristisches und Rhetorisches in Kanonistik und Legistik, Osnabrück, Universitätsverlag Rasch, 2000, 95-124.

Kaiser 2001, W. Kaiser, Schreiber und Korrektoren des Codex Florentinus, ZRG 118 (2001), 133-219.

Kaiser 2004, W. Kaiser, Die Epitome Iuliani. Beiträge zum römischen Recht im frühen Mittelalter und zum byzantinischen Rechtsunterricht, Frankfurt am Main, V. Klostermann, 2004.

Kaiser 2008, W. Kaiser, Nachvergleichungen von Novellen- und Codexzitaten in einer frühmittelalterlichen Sammlung mit Exzerpten aus dem Register Gregors d. Gr. (Reg. 13, 49 [50]), ZRG 125 (2008), 603-644.

Kaiser 2010, W. Kaiser, Zum Zeitpunkt des Inkrafttretens von Kaisergesetzen unter Justinian, ZRG 127 (2010), 172-201.

Kaiser 2013, W. Kaiser, Spätantike Rechtstexte in agrimensorischen Sammlungen, ZRG 130 (2013), 273-347.

Keenan 2014, L.G. Keenan, Law in the Byzantine period, in L.G. Keenan - J.G Manning - U. Yiftach-Firanko (eds.), Law and Legal Practice in Egypt from Alexander to the Arab Conquest. A Selection of Papyrological Sources in Translation, with Introduction and Commentary, Cambridge, Cambridge University Press, 2014, 23-28.

Lachmann 1848, K. Lachmann, Gromatici ueteres I = F. Blume - K. Lachmann - A. Rudorff, Die Schriften der römischen Feldmesser I. Texte und Zeichnungen, Berlin, G. Reimer, 1848, 276-280.

Lanata 1984, G. Lanata, Legislazione e natura nelle Novelle giustinianee, Napoli, Edizioni Scientifiche Italiane, 1984.

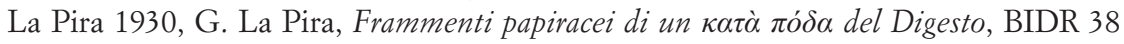
(1930), 151-174.

Lenel 1889, O. Lenel, Palingenesia iuris ciuilis II, Lipsiae, B. Tauchnitz, 1889.

Loschiavo 2014, L. Loschiavo, Insegnamento del diritto e cultura giuridica a Roma da Teoderico a Carlo Magno. La scia dei manoscritti, in G. Bassanelli Sommariva - S. Tarozzi (a cura di), Ravenna Capitale. Permanenze del mondo giuridico romano in Occidente nei secoli V-VIII: instrumenta, civitates, collegia, studium iuris, Santarcangelo di Romagna, Maggioli, 2014, 5-50.

Loschiavo 2015, L. Loschiavo, Was Rome still a Centre of Legal Culture between the $6^{\text {th }}$ and $8^{\text {th }}$ Centuries? Chasing the Manuscripts, Zeitschrift des Max-Planck-Instituts für europäische Rechtsgeschichte 23 (2015), 83-108.

Lowe 1925, E.A. Lowe, Some Facts about Our Oldest Latin Manuscripts, CQ 19 (1925), 197-208 = Id., Palaeographical Papers I, Oxford, Clarendon Press, 1972, 187 202.

Lowe 1961, E.A. Lowe, Greek Symptoms in a Sixth-Century Manuscript of St. Augustine and in a Group of Latin Legal Manuscripts, in S. Prete (ed.), Didascaliae. Studies in Honor of Anselm M. Albareda, Prefect of the Vatican Library, Presented by a 
Group of American Scholars, New York, B.M. Rosenthal, 1961, 279-289 = Id., Palaeographical Papers II, Oxford, Clarendon Press, 1972, 466-474.

Macino 2008, F. Macino, Sulle tracce delle Istituzioni di Giustiniano nell'alto medioevo. I manoscritti dal VI al XII secolo, Città del Vaticano, Biblioteca Apostolica Vaticana, 2008.

Maehler 2000, H. Maehler, L'évolution matérielle de l'bypomnèma jusqu'à la basse époque. Le cas du POxy. 856 (Aristophane) e PWürzburg 1 (Euripide), in M.-O. Goulet-Cazé (éd.), Le commentaire entre tradition et innovation (Actes du colloque international de l'Institut des traditions textuelles, Paris et Villejuif, 22-25 septembre 1999), Paris, Vrin, 2000, 29-36.

Mantovani 1987, D. Mantovani, Digesto e masse blubmiane, Milano, A. Giuffrè, 1987.

Mantovani 2007, D. Mantovani, Aggiornamenti sull'Appendix e i tempi di compilazione del Digesto, in Fides Humanitas Ius. Studii in onore di Luigi Labruna V, Napoli, Editoriale Scientifica, 2007, 3181-3203.

Mantovani 2015, D. Mantovani, Tituli e capita nelle Institutiones di Gaio e nell'Epitome Gai. Contributo allo studio del paratesto negli scritti dei giuristi romani, Seminarios Complutenses de Derecho Romano 28 (2015), 587-622.

Mantovani 2018, D. Mantovani, Les juristes écrivains de la Rome antique. Les ceuvres des juristes comme littérature, Paris, Collège de France - Les Belles Lettres, 2018.

McNamee 2007, K. McNamee, Annotations in Greek and Latin Texts from Egypt, Oakville, American Society of Papyrologists, 2007.

Menci 1997, G. Menci, L'impaginazione nel rotolo e nel codice, in B. Kramer - W. Luppe - H. Maehler - G. Poethke (hrsg.), Akten des 21. internationalen Papyrologenkongresses (Berlin, 13.-19. 8. 1995) II, Stuttgart-Leipzig, B.G. Teubner, 1997, 682-690.

Mommsen 1870, Th. Mommsen, Digesta Iustiniani Augusti I, Berolini, apud Weidmannos, 1870.

Naber 1935, J.C. Naber, Scholia ad Pandectas Graeca in integrum restituta tria, in P. Ciapessoni (a cura di), Studi in memoria di Aldo Albertoni I. Diritto romano e bizantino, Padova, CEDAM, 1935, 21-23.

Nagel 2014, P. Nagel, Codex apocryphus gnosticus Novi Testamenti I. Evangelien und Apostelgeschichten aus den Schriften von Nag Hammadi und verwandten Kodizes, Tübingen, Mohr Siebeck, 2014.

Palme 2007, B. Palme, The imperial presence: Government and army, in R.S. Bagnall (ed.), Egypt in the Byzantine World, 300-700, Cambridge, Cambridge University Press, 2007, 244-270.

Pandette 1902-1910, Justiniani Augusti digestorum seu pandectarum codex Florentinus olim Pisanus phototypice expressus, a cura della Commissione ministeriale per la riproduzione delle Pandette, Roma, Danesi, 1902-1910.

Pieler 2000, P.E. Pieler, Die justinianische Kodifikation in der juristischen Praxis des 6. Jabrbunderts, in S. Puliatti - A. Sanguinetti (a cura di), Legislazione, cultura giuridica, prassi dell'impero d'Oriente in età giustinianea tra passato e futuro (Atti del Convegno, Modena, 21-22 maggio 1998), Milano, A. Giuffrè, 2000, 211-227.

Puech 2006, V. Puech, Malalas et la prosopographie du VIe siècle: un éclairage sur le régime de Justinien, in S. Agusta-Boularot - J. Beaucamp (éds.), Recherches sur la 
Chronique de Jean Malalas II, Paris, Association des amis du Centre d'histoire et civilisation de Byzance, 2006, 213-226.

Porro 2011, A. Porro, P.Oxy. XX 2258 riconsiderato, Eikasmos 22 (2011), 311-332.

Puliatti 2013, S. Puliatti, "Arcaismi" come valori nella legislazione pubblicistica di Giustiniano, Iura 61 (2013), 180-197.

Roberts 1938, C.H. Roberts, Catalogue of the Greek and Latin Papyri in the John Rylands Library, Manchester III. Theological and Literary Texts (Nos. 457-551), Manchester, Manchester University Press, 1938.

Rotondi 1918, G. Rotondi, La codificazione giustinianea attraverso le fonti extragiuridiche, RISG 60 (1918), 239-268 = Id., Scritti giuridici I. Studii sulla storia delle fonti e sul diritto pubblico romano, Pavia, Tipografia e legatoria cooperativa, 1922, 340-369.

Scheltema 1958, H.J. Scheltema, Über die Werke des Stephanus, TRG 26 (1958), 5-14 = Id., Opera minora ad iuris historiam pertinentia, Groningue, Chimaira, 2004, 331-337.

Scheltema 1970, H.J. Scheltema, L'enseignement du droit des antécesseurs, Leiden, Brill, 1970 = Id., Opera minora ad iuris historiam pertinentia, Groningue, Chimaira, 2004, 58-110.

Schiller 1970, A.A. Schiller, The fate of imperial legislation in late Byzantine Egypt, in J.N. Hazard - W.J. Wagner (eds.), Legal Thought in the United States of America Under Contemporary Pressure. Reports from the United States of America on topics of major concern as established for the VIII Congress of the International Academy of Comparative Law, Brussels, Bruylant, 1970, 41-60.

Schiller 1971, A.A. Schiller, The courts are no more, in Studi in onore di Edoardo Volterra I, Milano, A. Giuffrè, 1971, 469-502.

Schoell-Kroll 1954, R. Schoell - G. Kroll, Nouellae, Berolini, Weidmann, $1954^{6}$.

Schulz 1941, F. Schulz, Fragmente des liber singularis de legatis?, TRG 17 (1941), 1927.

Scott 2012, R.D. Scott, Malalas and Justinian's Codification, in Id., Byzantine chronicles and the Sixth Century, Farnham-Burlington, Ashgate, 2012, 12-30.

Seider 1981, R. Seider, Paläographie der lateinischen Papyri II. Literarische Papyri 2. Juristische und christliche Texte, Stuttgart, A. Hiersemann, 1981.

Seidl 1954, E. Seidl, Die Jurisprudenz der ägyptischen Provinzialrichter byzantinischer Zeit, in W. Kunkel - H.J. Wolff (hrsg.), Festschrift für Ernst Rabel II. Geschichte der antiken Rechte und allgemeine Rechtslebre, Tübingen, Mohr, 1954, 235-261.

Seidl 1967, E. Seidl, Juristische Papyruskunde, SDHI 33 (1967), 503-580.

Simon 1988, D. Simon, Die Epochen der byzantinischen Rechtsgeschichte, Ius commune 15 (1988), 100-104.

Sirks-Sijpsteijn-Worp 1996, A.J.B. Sirks - P.J. Sijpsteijn - K.A. Worp, Ein frübbyzantinisches Szenario für die Amtswechslung in der Sitonie. Die griechischen Papyri aus Pommersfelden (PPG) mit einem Anhang über: die Pommersfeldener Digestenfragmente und die Überlieferungsgeschichte der Digesten, München, C.H. Beck, 1996.

Steinwenter 1952, A. Steinwenter, Was beweisen die Papyri für die praktische Geltung des justinianischen Gesetzgebungswerkes, Aegyptus 32 (1952), 131-137. 
Steinwenter 1958, A. Steinwenter, Aus dem kirchlichen Vermögensrechte der Papyri, ZRG KA 44 (1958), 1-34.

Stolte 1984, B.H. Stolte, The partes of the Digest in the Codex Florentinus, SGron 1 (1984), 69-91.

Stolte 1992, B.H. Stolte, Finium regundorum and the Agrimensores, SGron 5 (1992), 6172.

Stolte 1998, B.H. Stolte, Not new, but novel. Notes on the bistoriography of Byzantine law, Byzantine and Modern Greek Studies 22 (1998), 264-279.

Stolte 1999a, B.H. Stolte, Some thoughts on the early bistory of the Digest, SGron 6 (1999), 28103-119.

Stolte 1999b, B.H. Stolte, rec. A.J.B. Sirks - P.J. Sijpsteijn - K.A. Worp, Ein frübbyzantinisches Szenario für die Amtswechslung in der Sitonie. Die griechischen Papyri aus Pommersfelden (PPG) mit einem Anhang über: die Pommersfeldener Digestenfragmente und die Überlieferungsgeschichte der Digesten, München, C.H. Beck, 1996, TRG 67 (1999), 141-143.

Szirmai 1999, J.A. Szirmai, The Archaeology of Medieval Bookbinding, AldershotBurlington-Singapore-Sidney, Ashgate, 1999.

Taubenschlag 1940-1941, R. Taubenschlag, The Legislation of Justinian in the Light of the Papyri, Byzantion 15 (1940-1941), 280-295 = Id., Opera minora II. Spezieller Teil, Warszawa, Panstwowe Wydawnictwo Naukowe, 1959, 69-89.

Thulin 1913, C. Thulin, Corpus agrimensorum Romanorum I, 1. Opuscula agrimensorum ueterum, Lipsiae, B.G. Teubner, 1913.

Thurn 2000, I. [J.] Thurn, Ioannis Malalae chronographia, Berolini-Noui Eboraci, W. de Gruyter, 2000

Tjäder 1958, J.-O. Tjäder, Ein Verhandlungsprotokoll aus dem J. 433 n. Chr. (Pommersfelden, Papyrus lat. 14 r.), Scriptorium 12 (1958), 3-43.

Turner 1977, E.G. Turner, The Typology of the Early Codex, Philadelphia, University of Pennsylvania Press, 1977.

van der Wal 1979, N. van der Wal, Encore une fois le P. Reinach inv. 2173, TRG 47 (1979), 275-276.

van der Wal 1983, N. van der Wal, Die Schreibweise der dem lateinischen Entlehnten Fachworte in der frübbyzantinischen Juristensprache, Scriptorium 37 (1983), 29-53.

Watts, E. Watts, Justinian, Malalas, and the End of Athenian Philosophical Teaching in A.D. 529, JRS 94 (2004), 168-182.

Wenger 1950, L. Wenger, Aus fünfzig Jabren juristischer Papyrusforschung, in S. Morenz (hrsg.), Aus Antike und Orient. Festschrift Wilhelm Schubart zum 75. Geburtstag, Leipzig, Harrassowitz, 1950, 133-145.

Wenger 1953, L. Wenger, Die Quellen des Römischen Rechts, Wien, A. Holzhausens, 1953.

Zachariä 1842, [K.] E. Zachariä [von Lingenthal], Papyrusfragmente, Zeitschrift für die geschichtliche Rechtswissenschaft 11 (1842), 239-286. 